\title{
No-arbitrage under a class of honest times
}

\author{
Anna Aksamit • Tahir Choulli · Jun Deng • \\ Monique Jeanblanc
}

Received: 01 April 2016 / Accepted: 20 March 2017

\begin{abstract}
This paper quantifies the interplay between the no-arbitrage notion of nounbounded-profit-with-bounded-risk (NUPBR hereafter) and additional progressive information generated by a random time. This study complements the one of Aksamit et al. [2] in which the authors have studied similar topics for the case of stopping at the random time instead, while herein we deal with the part after the occurrence of the random time. Given that all the literature, up to our knowledge, proves that NUPBR is always violated after honest times that avoid stopping times in a continuous filtration, herein we propose a new class of honest times for which NUPBR can be preserved for some models. For these honest times, we obtain two principal results. The first result characterizes the pairs of initial market and honest time for which the resulting model preserves NUPBR, while the second result characterizes honest times that do not affect NUPBR of any quasi-left-continuous model (i.e., model in which the assets' price process has no predictable jump times). Furthermore, we construct explicitly local martingale deflators for a large class of models.
\end{abstract}

Mathematics Subject Classification (2010): 91B44, 91B70, 91G40, 91G99, 60G48, 60G51, 60G57, 60H99.

JEL Classification: D80, D81, D82, G14.

Anna Aksamit

Mathematical Institute, University of Oxford, Oxford, United Kingdom

Tahir Choulli (corresponding author)

Mathematical and Statistical Sciences Depart., University of Alberta, Edmonton, Canada

E-mail: tchoulli@ualberta.ca

Jun Deng

School of Banking and Finance, University of International Business and Economics, Beijing, China

Monique Jeanblanc

Laboratoire de Mathématiques et Modelisation d'Évry (LaMME), Université d'Evry Val d'Essonne, UMR

CNRS 8071, France 


\section{Introduction}

This paper complements the study undertaken in [2] about quantifying the exact interplay between an extra information/uncertainty and arbitrage for quasi-left-continuous models ${ }^{1}$. Similarly as in [2], we focus on the no-arbitrage concept of no-unboundedprofit-with-bounded-risk (NUPBR hereafter), and the extra information is the time of the occurrence of a random time. In the following section, we shall make precise the definition of NUPBR. It has been proved, in [11], that for models violating NUPBR, the numéraire portfolio does not exist and the maximisation utility problem does not have a solution. This solution can not exist even locally and/or under another equivalent probability measure and/or for very nice utilities. As a result, the utility indifference pricing/valuation rule fails and the benchmark approach developed by Platen in [23] can not be applicable. Thus, the market model violating the NUPBR is worthless financially and mathematically.

1.1 What are the main goals and the related literature?

Throughout the paper, we consider given a stochastic basis $(\Omega, \mathcal{G}, \mathbb{F}, P)$, where $\mathcal{F}_{\infty}:=\vee_{t \geq 0} \mathcal{F}_{t} \subseteq \mathcal{G}$, and the filtration $\mathbb{F}:=\left(\mathcal{F}_{t}\right)_{t \geq 0}$ satisfies the usual hypotheses (i.e., right continuity and completeness) and models the "public" information that all agents receive through time. On this basis, we suppose given a $d$-dimensional quasi-left-continuous semimartingale $S$ which represents the discounted assets' price process. Throughout the paper, the triplet $(S, \mathbb{F}, P)$ will be called the initial market model. In addition to this initial model, we consider a given random time (a non-negative random variable) denoted by $\tau$. This random time can represent the death time of an insurer, the default time of a firm, or any occurrence time of an influential event that can impact the market somehow. In this setting, our aim lies in answering the following.

$$
\text { If }(S, \mathbb{F}) \text { satisfies NUPBR, then what can be said about }(S, \mathbb{F}, \tau) \text { ? }
$$

After modeling the new informational system, this question translates into whether $(S, \mathbb{G})$ fullfils NUPBR or not. Here $\mathbb{G}$, that will be specified mathematically in the next section, is the new information that incorporates $\mathbb{F}$ and $\tau$ as soon as it occurs, and makes $\tau$ a $\mathbb{G}$-stopping time. Thanks to [24], see also [10] for the continuous case and [21] for the one dimensional case, one can easily prove that $(S, \mathbb{G})$ satisfies NUPBR if and only if both models $\left(S^{\tau}, \mathbb{G}\right)^{2}$ and $\left(S-S^{\tau}, \mathbb{G}\right)$ fulfill NUPBR. In [2] (see also [1] and [4] for related results), the authors focused on $\left(S^{\tau}, \mathbb{G}\right)$, while the second part $\left(S-S^{\tau}, \mathbb{G}\right)$ constitutes the main objective of the current paper.

As it will be mathematically specified later, NUPBR consists, roughly speaking, of "controlling" the gain processes that are bounded below by a given constant. Mathematically speaking, the gain processes are stochastic integrals with respect to the assets' price process in the Ito's sense. Thus, due to the Bichteler-DellacherieMokobodski theorem, see [13, Théorème 80, Chapitre VIII], the first obstacle in

\footnotetext{
1 A quasi-left-continuous model is a model in which the assets' price process does not jump on predictable stopping times

$2 S^{\tau}$ means as usual the process $S$ stopped at time $\tau$
} 
investigating NUPBR for $\left(S-S^{\tau}, \mathbb{G}\right)$ lies in whether the process $S-S^{\tau}$ is an integrator for general "admissible" (not only buy-and-hold) financial strategies or not. Or equivalently whether the model under consideration possesses the semimartingale property or not. Thus, the honesty assumption on $\tau$ guarantees the preservation of the semimartingale property after $\tau$. It is known that (see [20, Théorème 4.14]), in contrast to $\left(S^{\tau}, \mathbb{G}\right)$, the semimartingale property might fail for $\left(S-S^{\tau}, \mathbb{G}\right)$ when $\tau$ is arbitrary general. Therefore, for the rest of the paper, $\tau$ is assumed to be honest, a fact that will be mathematically defined in the next section. Recently, in [16] and [18], it is proved that NUPBR fails for $\left(S-S^{\tau}, \mathbb{G}\right)$ when $\tau$ is honest and avoids stopping times under Brownian filtration. For more works on this issue, we refer the reader to [3] and the references therein. Thus, our first goal is to answer the following

Is there any $\tau$ for which NUPBR is preserved for some markets?

In this paper, we answer this question positively, and we focus afterwards on quantifying the interplay between $\tau$ and the initial market model that impacts NUPBR after $\tau$. This latter objective can be achieved by singling out the part of $S$, denoted by $\mathcal{T}(S)$, that is truly relevant for NUPBR of $\left(S-S^{\tau}, \mathbb{G}\right)$. Or equivalently

$$
(\mathcal{T}(S), \mathbb{F}) \text { satisfies NUPBR if and only if }\left(S-S^{\tau}, \mathbb{G}\right) \text { does. }
$$

\subsection{Our financial and mathematical achievements}

Our first original contribution proposes a class of honest times for which there are markets that preserve NUPBR after $\tau$, and hence our aim described in (1.1) will be reached. Our family of honest times includes all the $\mathbb{F}$-stopping times as well as many examples of non $\mathbb{F}$-stopping times. By considering this subclass of honest times throughout the paper, our second novelty resides in answering (1.2), and describing as explicitly as possible the process $\mathcal{T}(S)$. As a result, if the honest time belongs to our class and the assets' price process $S$ is continuous, then $S-S^{\tau}$ fulfills NUPBR(G).

This paper is organized as follows. In the following section (Sect. 2), we present our main results, their immediate consequences, and/or their economic and financial interpretations. In this section, we also develop many examples and show how the main ideas come into play. Section 3 deals with the derivation of explicit local martingale deflators for a class of processes. The last section (Sect. 4) focuses on proving the main theorems and other related results. The paper contains also an appendix where some of existing and/or new technical results are summarized.

\section{The main results and their financial interpretations}

This section contains three subsections. The first subsection gives the notation, the definitions of NUPBR and honest times, and recalls their main properties. The second subsection develops simple examples of informational markets and explains how some ingredients of the main results play natural and important roles. The last subsection announces the principal results, their applications, and gives their financial meaning as well. 
2.1 Notation and preliminaries

As it is aforementioned, a filtered probability space $(\Omega, \mathcal{G}, \mathbb{F}, P)$ satisfying the usual conditions is given.

In what follows, $\mathbb{H}$ denotes a filtration satisfying the usual hypotheses, and $Q$ is a probability measure. The set of $\mathbb{H}$-martingales under $Q$ is denoted by $\mathcal{M}(Q, \mathbb{H})$. As usual, $\mathcal{A}^{+}(Q, \mathbb{H})$ is the set of increasing, right-continuous, $\mathbb{H}$-adapted and integrable processes under $Q$. If $\mathcal{C}(Q, \mathbb{H})$ is a class of $\mathbb{H}$-adapted processes, we denote by $\mathcal{C}_{\text {loc }}(Q, \mathbb{H})$ the set of processes $X$ such that there exists a sequence $\left(T_{n}\right)_{n \geq 1}$ of $\mathbb{H}$-stopping times that increases to $+\infty$ and the stopped processes $X^{T_{n}}$ belong to $\mathcal{C}(Q, \mathbb{H})$. Throughout the paper, when the probability is not mentioned, this means that we work under $Q=P$. Thus when $Q=P$, for simplicity, we write $\mathcal{M}(\mathbb{H})$, $\mathcal{A}(\mathbb{H})$, or in general $\mathcal{C}(\mathbb{H})$ and $\mathcal{C}_{\text {loc }}(\mathbb{H})$.

For a process $K$ with $\mathbb{H}$-locally integrable variation, we denote by $K^{o, \mathbb{H}}$ (respectively $K^{p, \mathbb{H}}$ ) its dual optional (respectively dual predictable) projection. For a process $X,{ }^{o, \mathbb{H}} X$ (respectively ${ }^{p, \mathbb{H}} X$ ) represents its optional (respectively predictable) projection with respect to $\mathbb{H}$.

To emphasize the role of the effect of filtration, we will denote $\langle., .\rangle^{\mathbb{F}}$, or $\langle., .\rangle^{\mathbb{G}}$ to specify the sharp bracket (predictable covariation process) calculated in the filtration $\mathbb{F}$ or $\mathbb{G}$, if confusion may rise. We recall that, for general semimartingales $X$ and $Y$, the sharp bracket is (if it exists) the dual predictable projection of the covariation process $[X, Y]$.

For a finite-dimensional $\mathbb{H}$-semimartingale $X$, the set $\mathcal{L}(X, \mathbb{H})$ is the set of $\mathbb{H}$-predictable processes having the same dimension as $X$ and being integrable with respect to $X$ and for $H \in \mathcal{L}(X, \mathbb{H})$, the resulting integral is the one-dimensional process denoted by $H \cdot X_{t}:=\int_{0}^{t} H_{s} d X_{s}$. Throughout the paper, stochastic processes have arbitrary finite dimension (in case it is not specified).

We recall the no-arbitrage notion that is addressed in this paper.

Definition 2.1 An $\mathbb{H}$-semimartingale $X$ satisfies the no-unbounded-profit-with-boundedrisk condition under $(\mathbb{H}, Q)$ if for all $T \in(0,+\infty)$, the set

$$
\mathcal{K}_{T}(X):=\left\{H \cdot X_{T}: H \in \mathcal{L}(X, \mathbb{H}), \text { and } H \cdot X \geq-1\right\}
$$

is bounded in probability under $Q$. Often, we abbreviate by saying $X$ satisfies $\operatorname{NUPBR}(\mathbb{H}, Q)$, or the model $(X, \mathbb{H}, Q)$ satisfies NUPBR. When $Q$ is equivalent to $P$, we suppress the probability in order to simplify the notation.

For more details about this no-arbitrage condition and its relationship with the literature, we refer the reader to [2]. NUPBR is intimately related to the existence of a $\sigma$-martingale density. Below, we recall the definitions of $\sigma$-martingale and $\sigma$-martingale density for a process.

Definition 2.2 An $\mathbb{H}$-adapted process $X$ is called an $(\mathbb{H}, \sigma)$-martingale if there exists a real-valued $\mathbb{H}$-predictable process $\phi$ such that

$$
0<\phi \leq 1, \quad \text { and } \phi \cdot X \text { is an } \mathbb{H} \text {-martingale. }
$$


A positive $\mathbb{H}$-local martingale $L$ is called an $(\mathbb{H}, \sigma)$-martingale density for an $\mathbb{H}$-adapted process $X$ (also called $\mathbb{H}$-deflator for $X$ ) if the product $L X$ is an $(\mathbb{H}, \sigma)$-martingale. The set of all $\mathbb{H}$-deflators for $X$ is denoted by

$$
\mathcal{L}_{\sigma}(X, \mathbb{H}):=\left\{L \in \mathcal{M}_{\text {loc }}(\mathbb{H}): L>0, L X \text { is an }(\mathbb{H}, \sigma) \text {-martingale }\right\} .
$$

The equivalence between NUPBR for $(X, \mathbb{H})$ and $\mathcal{L}_{\sigma}(X, \mathbb{H}) \neq \emptyset$ is established in $[2$, Proposition 2.3] when the horizon may be infinite, and in [24] for the case of finite horizon.

Beside the initial model $(S, \mathbb{F}, P)$ in which $S$ is assumed to be a quasi-leftcontinuous $\mathbb{F}$-semimartingale, we consider a random time $\tau$, i.e., random variable with values in $[0,+\infty]$, to which we associate the process $D$ and the filtration $\mathbb{G}$ given by

$$
D:=I_{\llbracket \tau,+\infty \llbracket}, \quad \mathbb{G}=\left(\mathcal{G}_{t}\right)_{t \geq 0}, \quad \mathcal{G}_{t}:=\bigcap_{s>t}\left(\mathcal{F}_{s} \vee \sigma\left(D_{u}, u \leq s\right)\right) .
$$

The filtration $\mathbb{G}$ is the smallest right-continuous filtration which contains $\mathbb{F}$ and makes $\tau$ a stopping time. In the literature, $\mathbb{G}$ is called the progressive enlargement of $\mathbb{F}$ with $\tau$. In addition to $\mathbb{G}$ and $D$, we associate to $\tau$ two important $\mathbb{F}$-supermartingales: the $\mathbb{F}$-optional projection of $I_{\llbracket 0, \tau \llbracket}$ denoted by $Z$, and the $\mathbb{F}$-optional projection of $I_{\llbracket 0, \tau \rrbracket}$, denoted by $\widetilde{Z}$, which satisfy

$$
Z_{t}:=P\left[\tau>t \mid \mathcal{F}_{t}\right] \text { and } \widetilde{Z}_{t}:=P\left[\tau \geq t \mid \mathcal{F}_{t}\right]
$$

The process $Z$ is right-continuous with left limits, while $\widetilde{Z}$ admits right and left limits. An important $\mathbb{F}$-martingale, denoted by $m$, is given by

$$
m:=Z+D^{o, \mathbb{F}},
$$

where $D^{o, \mathbb{F}}$ is the $\mathbb{F}$-dual optional projection of $D$. Obviously, $Z$ is bounded and $D^{o, \mathbb{F}}$ is nondecreasing and integrable.

For the reader's convenience, we recall the definition of honest time.

Definition 2.3 A random time $\sigma$ is called an $\mathbb{F}$-honest time, if for any $t$, there exists an $\mathcal{F}_{t}$-measurable r.v. $\sigma_{t}$ such that $\sigma I_{\{\sigma<t\}}=\sigma_{t} I_{\{\sigma<t\}}$.

We refer to Jeulin [20, Chapitre 5] and Barlow [9] for more information about honest times. In this paper, we restrict our study to the following subclass $\mathcal{H}$ of random times:

$$
\mathcal{H}:=\left\{\tau: \tau \text { is a finite } \mathbb{F} \text {-honest time satisfying } Z_{\tau}<1, P \text {-a.s. }\right\}
$$

Due to $Z_{\tau} I_{\{\tau<+\infty\}}=0, P$-a.s. when $\tau$ is an $\mathbb{F}$-stopping time, it is clear that all finite $\mathbb{F}$-stopping times belong to $\mathcal{H}$, and hence $\mathcal{H} \neq \varnothing$. In the case where $\mathbb{F}$ is the completed Brownian filtration, we can consider the $\mathbb{F}$-stopping times

$$
U_{0}^{\epsilon}=V_{0}^{\epsilon}=0, \quad U_{n}^{\epsilon}:=\inf \left\{t \geq V_{n-1}^{\epsilon}: B_{t}=\epsilon\right\}, \quad V_{n}^{\epsilon}:=\inf \left\{t \geq U_{n}^{\epsilon}: B_{t}=0\right\},
$$

where $0<\epsilon<1$ and $B$ is a one dimensional standard Brownian motion. Then

$$
\tau:=\sup \left\{V_{n}^{\epsilon}: V_{n}^{\epsilon} \leq T_{1}\right\},
$$


where $T_{1}:=\inf \left\{t \geq 0: B_{t}=1\right\}$, is an honest time which is not a stopping time, and belongs to $\mathcal{H}$ (see [6] for detailed proof). More examples of elements of $\mathcal{H}$ that are not stopping times are given in the next subsection. Furthermore, our class $\mathcal{H}$ bears the core idea behind the study in [5], where the authors show that $\mathcal{H}$ is very rich and plays an important role in the decomposition of random times. Furthermore, in the case of discrete time market models, the set $\mathcal{H}$ coincides with the whole set of finite honest times.

Remark 2.4 (a) If $\tau$ avoids $\mathbb{F}$-stopping times, then $Z_{\tau}=1$.

(b) If $\tau$ is honest and avoids $\mathbb{F}$ stopping times, and if there exists a continuous $\mathbb{F}$-martingale $S$ that enjoys predictable representation property, then $Z_{\tau}=1$, and $S-S^{\tau}$ fails NUPBR (see [16, Proposition 6.3]).

We now recall a lemma obtained in [2, Lemma 2.6].

Lemma 2.5 Let $X$ be an $\mathbb{H}$-predictable process with finite variation. Then $X$ satisfies $N U P B R(\mathbb{H})$ if and only if $X \equiv X_{0}$ (i.e., the process $X$ is constant).

We end this section with the following lemma which gives the $\mathbb{G}$-semimartingale decomposition of an $\mathbb{F}$-martingale.

Lemma 2.6 The following assertions hold.

(a) Let $M$ be an $\mathbb{F}$-local martingale, and $\tau$ be an honest time. Then the process $\widehat{M}$, defined as

$$
\widehat{M}:=M-M^{\tau}+\left(1-Z_{-}\right)^{-1} I_{\rrbracket \tau,+\infty \mathbb{I}} \cdot\langle M, m\rangle^{\mathbb{F}},
$$

is a $\mathbb{G}$-local martingale.

(b) If $\tau$ is an honest time such that $Z_{\tau} I_{\{\tau<+\infty\}} P$-a.s. (a fortiori if $\tau \in \mathcal{H}$ ), then the $\mathbb{G}$-predictable process $\left(1-Z_{-}\right)^{-1} I_{\rrbracket} \tau,+\infty \llbracket$ is $\mathbb{G}$-locally bounded.

Proof Assertion (a) is a standard result on progressive enlargement of filtration with honest times. For details, we refer the reader to [20, Théorème $(5,10)]$, [9] and [14, Chapter XX, Sect. 79].

Herein we prove assertion (b). It is known, see [14, Chapter XX, Sect. 79], that $Z=\widetilde{Z}$ on $\rrbracket \tau,+\infty \llbracket$, and

$$
\rrbracket \tau,+\infty \llbracket \subset\left\{Z_{-}<1\right\} \cap\{\widetilde{Z}<1\} \subset\left\{Z_{-}<1\right\} \cap\{Z<1\} .
$$

Due to $Z_{\tau} I_{\{\tau<+\infty\}} P$-a.s., we have $\llbracket \tau,+\infty \llbracket \subset\{Z<1\}$, and hence the process

$$
X:=(1-Z)^{-1} I_{\llbracket \tau,+\infty \llbracket}
$$

is càdlàg, $\mathbb{G}$-adapted and takes values in $[0,+\infty)$.

Combining these with $\rrbracket \tau,+\infty \llbracket \subset\left\{Z_{-}<1\right\}$, we can easily prove that

$$
T_{n}:=\inf \left\{t \geq 0: X_{t} \geq n\right\} \uparrow+\infty \text { and } \max \left(X^{T_{n}-}, X_{-}^{T_{n}}\right) \leq n, P \text {-a.s., }
$$

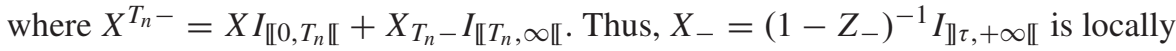
bounded, and the proof of the lemma is completed. 


\subsection{Particular cases and examples}

In this subsection, by analysing particular cases and examples, we obtain some results that are vital for understanding the exact interplay between the features of the initial market and the honest time under consideration. The following simple lemma plays a key role in this analysis.

Theorem 2.7 Suppose that $\tau \in \mathcal{H}$. If $S$ is continuous and satisfies $N U P B R(\mathbb{F})$, then $S-S^{\tau}$ satisfies $\operatorname{NUPBR}(\mathbb{G})$.

Proof Let $S=\left(S^{1}, \ldots, S^{d}\right)$ be a $d$-dimensional continuous process satisfying NUPBR $(\mathbb{F})$ Then there exists a positive $\mathbb{F}$-local martingale $L$ such that $L S$ is an $(\mathbb{F}, \sigma)$-martingale. Since $S$ is continuous and $L$ is a local martingale, we deduce that the increasing process $\sup _{u<.}\left|S_{u}\right| \sup _{u<.}\left|\Delta L_{u}\right|$ is locally integrable.

Thus, thanks to [7, Proposition 3.3] and

$$
\sum_{i=1}^{d} \Delta\left(L S^{i}\right)=\sum_{i=1}^{d} S^{i} \Delta L \geq-d \sup _{u \leq .}\left|S_{u}\right| \sup _{u \leq .}\left|\Delta L_{u}\right|,
$$

we conclude that $L S$ is an $\mathbb{F}$-local martingale. We consider a sequence of $\mathbb{F}$-stopping times $\left(T_{n}\right)_{n \geq 1}$ that increases to infinity such that both $L^{T_{n}}$ and $L^{T_{n}} S^{T_{n}}$ are martingales, and put $Q_{n}:=\left(L_{T_{n}} / L_{0}\right) \cdot P$ which is equivalent to $P$. Then on the one hand $S^{(n)}:=S^{T_{n}}$ is an $\left(\mathbb{F}, Q_{n}\right)$-martingale. On the other hand, in virtue of Proposition A.4, $S-S^{\tau}$ satisfies NUPBR $(\mathbb{G})$ if and only if $S^{(n)}-\left(S^{(n)}\right)^{\tau}=\left(S-S^{\tau}\right)^{T_{n}}$ satisfies NUPBR(G) under $Q_{n}$, for all $n \geq 1$. This shows that, without loss of generality, one only needs to prove the theorem when $S$ is an $\mathbb{F}$-martingale.

Therefore, for the rest of the proof, we assume that $S$ is an $\mathbb{F}$-martingale. Thanks to Lemma 2.6, the process $Y^{\mathbb{G}}:=\mathcal{E}\left(\left(1-Z_{-}\right)^{-1} I_{\rrbracket},+\infty \mathbb{} \cdot \widehat{m^{c}}\right)$ is a well-defined continuous real-valued and positive $\mathbb{G}$-local martingale, where $m^{c}$ is the continuous $\mathbb{F}$-local martingale part of $m$, and $\widehat{m^{c}}$ is defined as in (2.2). Thanks to the continuity of $S$ and (2.2) applied to $S$, we get

$$
\begin{aligned}
S-S^{\tau}+\left[S-S^{\tau}, \frac{I_{\rrbracket \tau,+\infty \llbracket}}{1-Z_{-}} \cdot \widehat{m^{c}}\right] & =S-S^{\tau}+\left(1-Z_{-}\right)^{-1} I_{\rrbracket \tau,+\infty \llbracket} \cdot\langle S, m\rangle^{\mathbb{F}} \\
& =\widehat{S} \in \mathcal{M}_{\mathrm{loc}}(\mathbb{G}) .
\end{aligned}
$$

Therefore, a combination of this and integration by parts formula applied to $\left(S-S^{\tau}\right) Y^{\mathbb{G}}$, we conclude that this latter process is a $\mathbb{G}$-local martingale. This proves $\operatorname{NUPBR}(\mathbb{G})$ for $S-S^{\tau}$, and the proof of the theorem is achieved.

Remark 2.8 Theorem 2.7 follows also from one of our principal results stated in the next subsection. However, due to the simplicity of the proof of this theorem, we have opted for detailing this proof above.

Theorem 2.7 asserts clearly that, when $\tau \in \mathcal{H}$, the model $\left(S-S^{\tau}, \mathbb{G}\right)$ might fail NUPBR only if $S$ jumps. Thus, the following natural question arises:

Does the condition $\{\Delta S \neq 0\} \cap \llbracket \tau \rrbracket=\emptyset$ impact $\operatorname{NUPBR}(\mathbb{G})$ ? 
Example 2.9 Suppose that $\mathbb{F}$ is generated by a Poisson process $N$ with intensity equal to 1 . Consider two real numbers $a>0$ and $\mu>1$, and set

$$
\tau:=\sup \left\{t \geq 0: Y_{t}:=\mu t-N_{t} \leq a\right\}, \quad \text { and } \quad M_{t}:=N_{t}-t .
$$

It can be proved easily, see [6, Proposition 4], that $\tau \in \mathcal{H}$, and the associated processes $Z$ and $\widetilde{Z}$ are given by

$$
Z=\Psi(Y-a) I_{\{Y \geq a\}}+I_{\{Y<a\}} \text { and } \widetilde{Z}=\Psi(Y-a) I_{\{Y>a\}}+I_{\{Y \leq a\}} .
$$

Here $\Psi(u):=P\left[\sup _{t \geq 0} Y_{t}>u\right]$ is the ruin probability associated to the process $Y$. As a result we have

$$
1-Z_{-}=\left(1-\Psi\left(Y_{-}-a\right)\right) I_{\left\{Y_{-}>a\right\}},
$$

and we can prove that $m=m_{0}+\phi \cdot M$, where

$$
\phi:=\left(\Psi\left(Y_{-}-a-1\right)-\Psi\left(Y_{-}-a\right)\right) I_{\left\{Y_{-}>1+a\right\}}+\left(1-\Psi\left(Y_{-}-a\right)\right) I_{\left\{a<Y_{-} \leq 1+a\right\}} .
$$

We define $S:=I_{\left\{a \leq Y_{-}<a+1\right\}} \cdot M$. Thanks to Lemma 2.6 and Itô's lemma, and by putting $V_{t}=t$ and letting $\widehat{S}$ be the $\mathbb{G}$-local martingale part of $S-S^{\tau}$, we derive

$$
\begin{aligned}
{[\widehat{S}, \widehat{S}] } & =I_{\rrbracket \tau,+\infty \llbracket} \cdot[S]=I_{\rrbracket \tau,+\infty \llbracket} \cdot S+I_{\left\{a<Y_{-}<a+1\right\}} I_{\rrbracket \tau,+\infty \llbracket \mathbb{I}} \cdot V \\
& =\widehat{S}+I_{\left\{a<Y_{-}<a+1\right\}} I_{\rrbracket \tau,+\infty \llbracket}\left(1-\frac{\phi}{1-Z_{-}}\right) \cdot V, \\
& =\widehat{S} \text { is a } \mathbb{G} \text {-local martingale. }
\end{aligned}
$$

The last equality is due to $\phi \equiv 1-Z_{-}$on $\left\{a<Y_{-}<a+1\right\}$. This proves that $\widehat{S} \equiv 0$, and hence $S-S^{\tau}$ is $\mathbb{G}$-predictable with finite variation. Thus, in virtue of Lemma 2.5, the process $S-S^{\tau}$ (which is not null) violates $\operatorname{NUPBR}(\mathbb{G})$. Therefore $(S, \mathbb{F})$ satisfies NUPBR, while $\left(S-S^{\tau}, \mathbb{G}\right)$ violates it.

Example 2.10 Consider the same setting and notation as in Example 2.9, except for $S$ that we suppose having the form $S=I_{\left\{Y_{-}>a+1\right\}} \cdot M$ instead. By combining Lemma 2.6, Itô's lemma and similar calculation as in (2.6), we deduce that both $Y^{\mathbb{G}}:=\mathcal{E}(\xi \cdot \widehat{S})$ and $Y^{\mathbb{G}}\left(S-S^{\tau}\right)$ are $\mathbb{G}$-local martingales and $Y^{\mathbb{G}}>0$. Here $\xi$ is given by

$$
\xi:=\frac{\Psi\left(Y_{-}-a-1\right)-\Psi\left(Y_{-}-a\right)}{1-\Psi\left(Y_{-}-a-1\right)} I_{\left\{Y_{-}>a+1\right\}} I_{\rrbracket \tau,+\infty \mathbb{I}},
$$

and as usual $\mathcal{E}(X)$ denotes the Doléans-Dade exponential of a semimartingale $X$. This proves that both models $(S, \mathbb{F})$ and $\left(S-S^{\tau}, \mathbb{G}\right)$ satisfy NUPBR.

Examples 2.9 and 2.10 can be encountered in the following financial context: The random time given by (2.4) represents the last time the cash reserve of a firm does not exceed the level $a$. Then Example 2.9 (respectively Example 2.10) corresponds to the case when the security asset's price process lives on $\left\{a \leq Y_{-}<1+a\right\}$ (respectively on $\left\{Y_{-}>1+a\right\}$ ). Remark that, in both Examples 2.9 and 2.10, the graph of the random time $\tau$ is included in a countable union of graphs of predictable stopping times. Hence, due to the quasi-left-continuity of $S$, we immediately conclude that $\{\Delta S \neq 0\} \cap \llbracket \tau \rrbracket$ is evanescent for both examples. This gives a negative answer to (2.3). 
2.3 Main results and their applications

Our first main result requires the following easy and interesting lemma.

Lemma 2.11 Suppose that $\tau \in \mathcal{H}$. Then the $\mathbb{F}$-adapted process

$$
V^{\mathbb{F}}:=\sum I_{\left\{\widetilde{Z}=1>Z_{-}\right\}}
$$

is càdlàg with finite values, and hence is $\mathbb{F}$-locally integrable.

Proof Thanks to Proposition B.1-(b), there exists a sequence of $\mathbb{F}$-stopping times, $\left(\sigma_{n}\right)_{n \geq 1}$ that increases to infinity almost surely and $1 \leq n^{2}\left(1-Z_{t-}\right)^{2}$ on $\left\{Z_{t-}<1\right\} \cap\left\{t \leq \sigma_{n}\right\}$ Thus, for any nonnegative and bounded $\mathbb{F}$-optional process $H$, we have

$$
\begin{aligned}
\left(H \cdot V^{\mathbb{F}}\right)^{\sigma_{n}} & \leq n^{2}\left(\sum H\left(1-Z_{-}\right)^{2} I_{\left\{\widetilde{Z}=1>Z_{-}\right\}}\right)^{\sigma_{n}} \\
& =n^{2}\left(\sum H(\Delta m)^{2} I_{\left\{\widetilde{Z}=1>Z_{-}\right\}}\right)^{\sigma_{n}} \leq n^{2}(H \cdot[m, m])^{\sigma_{n}} .
\end{aligned}
$$

Therefore, the proof of the lemma follows immediately by combining the above inequality and the fact that $[m, m] \in \mathcal{A}_{\text {loc }}^{+}(\mathbb{F})$.

In the following theorem, we announce our first main result, which is based on the following aforementioned transformation (see (1.2))

$$
\mathcal{T}(S):=S-\left[S, V^{\mathbb{F}}\right]=S-\sum I_{\left\{\widetilde{Z}=1>Z_{-}\right\}} \Delta S
$$

Theorem 2.12 Suppose that $S$ is an $\mathbb{F}$-quasi-left-continuous semimartingale, and $\tau \in \mathcal{H}$. Then the following are equivalent.

(a) $S-S^{\tau}$ satisfies $N U P B R(\mathbb{G})$.

(b) $\left(1-Z_{-}\right) \cdot \mathcal{T}(S)$ satisfies $N U P B R(\mathbb{F})$.

(c) $I_{\left\{Z_{-}<1\right\}} \cdot \mathcal{T}(S)$ satisfies $N U P B R(\mathbb{F})$.

Thanks to Proposition A.4, by considering $\phi:=1-Z_{-}+I_{\left\{Z_{-}=1\right\}}$ (which is $\mathbb{F}$-predictable and satisfies $0<\phi \leq 1$ ), we deduce the following fact: for any $\mathbb{F}$-semimartingale $X, I_{\left\{Z_{-}<1\right\}} \cdot X$ satisfies NUPBR(F) if and only if $\left(1-Z_{-}\right) \cdot X$ does. Therefore, the proof of (b) $\Longleftrightarrow$ (c) follows immediately from this fact. The implication (b) $\Longrightarrow$ (a) of the theorem can be stated in a more general form. Thus, the proof of this implication will be given, as a direct consequence of Corollary 3.5, at the end of Sect. 3. The implication (a) $\Longrightarrow$ (b) is the most technical and difficult part of the theorem. Thus, its proof is delegated to Subsection 4.2.

Corollary 2.13 Suppose that $S$ is $\mathbb{F}$-quasi-left-continuous, and $\tau \in \mathcal{H}$. Then the following assertions hold:

(a) If the pair $\left(S, \sum I_{\left\{\widetilde{Z}=1>Z_{-}\right\}} \Delta S\right)$ satisfies $N U P B R(\mathbb{F})$, then $S-S^{\tau}$ satisfies $N U P B R(\mathbb{G})$.

(b) If $S$ satisfies $N U P B R(\mathbb{F})$ and $\{\Delta S \neq 0\} \cap\left\{\widetilde{Z}=1>Z_{-}\right\}=\emptyset$, then $S-S^{\tau}$ satisfies $\operatorname{NUPBR}(\mathbb{G})$. 
Proof The assertion (a) follows by combining Theorem 2.12 and the equality

$$
\left(1-Z_{-}\right) \cdot \mathcal{T}(S)=\left(1-Z_{-},-\left(1-Z_{-}\right)\right) \cdot\left(S, \sum I_{\left\{\widetilde{Z}=1>Z_{-}\right\}} \Delta S\right) .
$$

Due to $\{\Delta S \neq 0\} \cap\left\{\widetilde{Z}=1>Z_{-}\right\}=\emptyset$, assertion (b) follows immediately from assertion (a), and the proof of the corollary is completed.

Remark 2.14 To illustrate Theorem 2.12 in the setting of Examples 2.9 and 2.10, we need to calculate $\left\{\widetilde{Z}=1>Z_{-}\right\}$. Due to $(2.5)$ and the equation that precedes it, we remark that $\left\{\widetilde{Z}=1>Z_{-}\right\}=\left\{a<Y_{-} \leq a+1\right\} \cap\{\Delta N \neq 0\}$.

For the model $(S, \mathbb{F})$ of Example 2.9, we derive

$$
I_{\left\{\widetilde{Z}=1>Z_{-}\right\}} \Delta S=I_{\left\{a \leq Y_{-}<a+1\right\}} I_{\left\{a<Y_{-} \leq a+1\right\}} \Delta N=I_{\left\{a<Y_{-}<a+1\right\}} \Delta N .
$$

This implies that, for this model,

$$
\mathcal{T}(S)=S-\sum I_{\left\{\widetilde{Z}=1>Z_{-}\right\}} \Delta S=I_{\left\{Y_{-}=a\right\}} \cdot M-\int_{0}^{\cdot} I_{\left\{a<Y_{S-}<a+1\right\}} d s .
$$

It is clear that $\mathcal{T}(S)$ has no $\sigma$-martingale density since the drift part of $\mathcal{T}(S)$ is not absolutely continuous -as a measure of time- with respect to $\langle\mathcal{T}(S)\rangle^{\mathbb{F}}$. Hence, a fortiori, $I_{\left\{Z_{-}<1\right\}} \cdot \mathcal{T}(S)=-\int_{0}^{\cdot} I_{\left\{a<Y_{s-}<a+1\right\}} d s$ violates $\operatorname{NUPBR}(\mathbb{F})$.

For the case of Example 2.10, we easily derive

$$
I_{\left\{\widetilde{Z}=1>Z_{-}\right\}} \Delta S=I_{\left\{Y_{-}>a+1\right\}} I_{\left\{a<Y_{-} \leq a+1\right\}} \Delta N \equiv 0 .
$$

Thus, this example falls into the case of Corollary 2.13-(b), and $\mathcal{T}(S) \equiv S$ satisfies $\operatorname{NUPBR}(\mathbb{F})$.

As application of Theorem 2.12 to Lévy models, we state the following

Theorem 2.15 Suppose that $\tau$ belongs to $\mathcal{H}$, and $S$ is $\mathbb{F}$-quasi-left-continuous. Let $\mu$ be the optional random measure associated to the jumps of $S$, and $\nu^{\mathbb{F}}$ (respectively $v^{\mathbb{G}}$ ) be the $\mathbb{F}$-compensator (respectively the $\mathbb{G}$ compensator) of $\mu$ (respectively of $\left.I_{\rrbracket \tau,+\infty \llbracket} \star \mu\right)$. If $S$ satisfies $N U P B R(\mathbb{F})$ and

$$
I_{\rrbracket \tau,+\infty \llbracket} \star v^{\mathbb{F}} \text { is equivalent to } v^{\mathbb{G}} P \text {-a.s., }
$$

then $S-S^{\tau}$ satisfies $N U P B R(\mathbb{G})$.

The proof of this theorem follows from Theorem 2.12 as long as we prove that, under (2.9), if $S$ satisfies NUPBR $(\mathbb{F})$, then $\mathcal{T}(S)$ satisfies NUPBR($(\mathbb{F})$. This proof is technical, and thus it is delegated to Sect. 4.

Remark 2.16 Remark that the absolute continuity $v^{\mathbb{G}} \ll I_{\rrbracket},+\infty \llbracket{ } \star v^{\mathbb{F}}, P$-a.s. holds. This follows from the fact that $v^{\mathbb{G}}$ is absolutely continuous with respect to $v^{\mathbb{F}}$ and it lives on $\rrbracket \tau,+\infty \llbracket$ only.

The Lévy case: If $S$ is a Lévy process and $F(d x)$ is its Lévy measure under $\mathbb{F}$, then $v^{\mathbb{F}}(d t, d x)=F(d x) d t$ and $v^{\mathbb{G}}(d t, d x)=I_{\{t>\tau\}} F_{t}^{\mathbb{G}}(d x) d t$, where $F_{t}^{\mathbb{G}}(d x)$ is its Lévy measure under $\mathbb{G}$. Thus, Theorem 2.15 asserts that if $P \otimes \lambda$ almost every 
$(\omega, t)(\lambda(d t)=d t), F_{t}^{\mathbb{G}}(\omega, d x)=f(t, x, \omega) F(d x)$ for some real-valued and positive functional $f(t, x, \omega)$, then $S-S^{\tau}$ satisfies $\operatorname{NUPBR}(\mathbb{G})$. For more examples of Lévy framework, we refer the reader to [15].

Examples 2.9-2.10 versus Theorem 2.15: In the context of Example 2.9, we easily calculate $v^{\mathbb{F}}(d t, d x)=I_{\left\{a<Y_{t-} \leq a+1\right\}} \delta_{1}(d x) d t$ and establish that

$$
v^{\mathbb{G}}(d t, d x)=I_{\{t>\tau\}} I_{\left\{a<Y_{t-} \leq a+1\right\}}\left(1-\phi_{t} /\left(1-Z_{t-}\right)\right) \delta_{1}(d x) d t \equiv 0
$$

which is not equivalent to $I_{\rrbracket \tau},+\infty \llbracket \star \nu^{\mathbb{F}}$. This example shows that (2.9) can be violated. Therefore, in those circumstances, we can not conclude whether $S-S^{\tau}$ satisfies $\operatorname{NUPBR}(\mathbb{G})$ or not directly from Theorem 2.15 .

For the case of Example 2.10, we have $v^{\mathbb{F}}(d t, d x)=I_{\left\{Y_{t->}>+1\right\}} \delta_{1}(d x) d t$ and $\nu^{\mathbb{G}}(d t, d x)=I_{\{t>\tau\}} I_{\left\{Y_{t->}>a+1\right\}}\left(1-\phi_{t} /\left(1-Z_{t-}\right)\right) \delta_{1}(d x) d t$ which is equivalent to $I_{\rrbracket \tau,+\infty \mathbb{I}} \star v^{\mathbb{F}}$ since $\left\{Y_{-}>a+1\right\} \subset\left\{\phi<1-Z_{-}\right\} P \otimes d t$-a.e. Thus, Theorem 2.15 allows us to conclude that $S-S^{\tau}$ fulfills $\operatorname{NUPBR}(\mathbb{G})$.

The rest of this subsection describes models of $\tau$ that do not affect NUPBR.

Theorem 2.17 Suppose $\tau \in \mathcal{H}$. Then the following assertions are equivalent.

(a) The set $\left\{\widetilde{Z}=1>Z_{-}\right\}$is accessible (i.e., it is contained in a countable union of graphs of $\mathbb{F}$-predictable stopping times).

(b) For every (bounded) $\mathbb{F}$-quasi-left-continuous martingale $X$, the process $X-X^{\tau}$ satisfies $\operatorname{NUPBR}(\mathbb{G})$.

(b') For any probability $Q$ equivalent to $P$ and every (bounded) $\mathbb{F}$-quasi-left-continuous $X \in \mathcal{M}(Q, \mathbb{F})$, the process $X-X^{\tau}$ satisfies $N U P B R(\mathbb{G})$.

(c) For every (bounded) $\mathbb{F}$-quasi-left-continuous process $X$ satisfying $N U P B R(\mathbb{F})$, the process $X-X^{\tau}$ satisfies $N U P B R(\mathbb{G})$.

Proof The proof of the theorem is organized in three parts, where we prove (a) $\Longleftrightarrow$ (b) (b) $\Longleftrightarrow$ (b') and (b') $\Longleftrightarrow$ (c) respectively.

Part 1: We start by proving that (a) implies (b). Suppose that the thin $\operatorname{set}\left\{\widetilde{Z}=1>Z_{-}\right\}$ is accessible. Then for any $\mathbb{F}$-quasi-left-continuous martingale $X$, we have $\{\Delta X \neq 0\} \cap\left\{\widetilde{Z}=1>Z_{-}\right\}=\emptyset$. Thanks to Corollary 2.13-(b), we deduce that $X-X^{\tau}$ satisfies $\operatorname{NUPBR}(\mathbb{G})$. This completes the proof of (a) $\Rightarrow(b)$. To prove the reverse, assuming that assertion (b) holds, we consider a sequence of stopping times $\left(T_{n}\right)_{n \geq 1}$ that exhausts the thin set $\left\{\widetilde{Z}=1>Z_{-}\right\}$, i.e., $\left\{\widetilde{Z}=1>Z_{-}\right\}=\bigcup_{n=1}^{+\infty} \llbracket T_{n} \rrbracket$. Then, each $T_{n}-$ that we denote by $T$ for the sake of simplicity- can be decomposed into a totally inaccessible part $T^{i}$ and an accessible part $T^{a}$ as $T=T^{i} \wedge T^{a}$. Consider the quasi-left-continuous $\mathbb{F}$-martingale

$$
M:=V-V^{p, \mathbb{F}}=: V-\widetilde{V},
$$

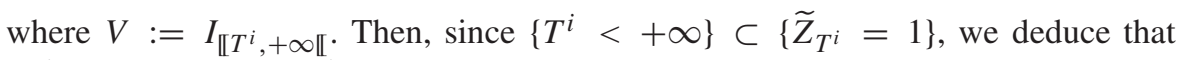
$\left\{T^{i}<+\infty\right\} \subset\left\{\tau \geq T^{i}\right\}$ and

$$
M-M^{\tau}=I_{\rrbracket \tau,+\infty \mathbb{I}} \cdot M=-I_{\rrbracket \tau,+\infty \llbracket} \cdot \widetilde{V} \text { is } \mathbb{G} \text {-predictable. }
$$

The finite variation and $\mathbb{G}$-predictable process, $I_{\rrbracket},+\infty \mathbb{I} \cdot M$, satisfies $\operatorname{NUPBR}(\mathbb{G})$. Hence, it is null, or equivalently

$$
0=E\left[I_{\rrbracket \tau,+\infty \mathbb{I}} \cdot \widetilde{V}_{\infty}\right]=E\left[\int_{0}^{\infty}\left(1-Z_{s_{-}}\right) d \widetilde{V}_{s}\right]=E\left[\left(1-Z_{T^{i-}}\right) I_{\left\{T^{i}<+\infty\right\}}\right] .
$$


Therefore, we conclude that $T^{i}=+\infty, P$-a.s., and the stopping time $T=T^{a}$ is accessible. This ends the proof of the equivalence between (a) and (b).

Part 2: It is easy to see that the implication (b') $\Longrightarrow$ (b) follows by taking $Q=P$. To prove the reverse sense, we suppose that a probability measure $Q$ equivalent to $P$ and an $\mathbb{F}$-quasi-left-continuous $X \in \mathcal{M}(Q, \mathbb{F})$ are given. Put

$Z_{t}^{\mathbb{F}}:=E\left[\frac{d Q}{d P} \mid \mathcal{F}_{t}\right]=: \mathcal{E}_{t}(N), \quad Y:=\left(\begin{array}{l}\mathcal{E}\left(N^{(q c)}\right) X \\ \mathcal{E}\left(N^{(q c)}\right)\end{array}\right)$ and $N^{(q c)}:=N-I_{\cup_{n}} \llbracket \sigma_{n} \rrbracket \mid N$,

where $\left(\sigma_{n}\right)_{n}$ is a sequence of $\mathbb{F}$-predictable stopping times that exhausts all the predictable jumps of $N$. In other words, $N^{(q c)}$ is the $\mathbb{F}$-quasi-left-continuous local martingale part of $N$, see [17, Theorem 7.25, Chapter VII] about local martingale decomposition. Then due to the quasi-left-continuity of $X$, simple calculations show that $Y$ is an $\mathbb{F}$-quasi-left-continuous martingale. Therefore, by applying directly assertion (b) to $Y$, we conclude that

$$
Y-Y^{\tau}=\left(\begin{array}{l}
\mathcal{E}\left(N^{(q c)}\right)\left(X-X^{\tau}\right)+X^{\tau}\left(\mathcal{E}\left(N^{(q c)}\right)-\mathcal{E}\left(N^{(q c)}\right)^{\tau}\right) \\
\mathcal{E}\left(N^{(q c)}\right)-\mathcal{E}\left(N^{(q c)}\right)^{\tau}
\end{array}\right)
$$

satisfies NUPBR $(\mathbb{G})$. Then, there exists a real-valued positive $\mathbb{G}$-local martingale $Z^{\mathbb{G}}$ such that $Z^{\mathbb{G}} \mathcal{E}\left(N^{(q c)}\right)\left(X-X^{\tau}\right)$ and $Z^{\mathbb{G}} \mathcal{E}\left(N^{(q c)}\right) / \mathcal{E}\left(N^{(q c)}\right)^{\tau}$ are both $\sigma$-martingales under $(\mathbb{G}, P)$. Since the process $Z^{\mathbb{G}} \mathcal{E}\left(N^{(q c)}\right) / \mathcal{E}\left(N^{(q c)}\right)^{\tau}$ is positive and thanks to Proposition 3.3 and Corollary 3.5 of [7] (which states that a non-negative $\sigma$-martingale is a local martingale), we deduce that $Z^{\mathbb{G}} \mathcal{E}\left(N^{(q c)}\right) / \mathcal{E}\left(N^{(q c)}\right)^{\tau}$ is a real-valued positive element of $\mathcal{M}_{\text {loc }}(\mathbb{G})$ such that $Z^{\mathbb{G}} \mathcal{E}\left(N^{(q c)}\right)\left(X-X^{\tau}\right)$ (or equivalently $Z^{\mathbb{G}} \mathcal{E}\left(N^{(q c)}\right)\left(X-X^{\tau}\right) / \mathcal{E}\left(N^{(q c)}\right)^{\tau}$ is a $\sigma$-martingale. This proves that $X-X^{\tau}$ satisfies $\operatorname{NUPBR}(\mathbb{G})$, and the proof of (b) $\Longleftrightarrow\left(b^{\prime}\right)$ is completed.

Part 3: Remark that $(\mathrm{c}) \Longrightarrow\left(\mathrm{b}^{\prime}\right)$ is obvious, and hence we focus on proving the reverse. Suppose that assertion (b') holds, and consider an $\mathbb{F}$-quasi-left-continuous process $X$ satisfying NUPBR $(\mathbb{F})$. Then there exists a real-valued and positive $\mathbb{F}$-local martingale $Y$, and a real-valued and $\mathbb{F}$-predictable process $\phi$ such that

$$
0<\phi \leq 1 \text { and } Y(\phi \cdot X) \text { is an } \mathbb{F} \text {-martingale. }
$$

Let $\left(T_{n}\right)$ be a sequence of $\mathbb{F}$-stopping times that increases to infinity (almost surely) such that $Y^{T_{n}}$ is a martingale, and set

$$
\bar{X}:=\phi \cdot X, \quad Q_{n}:=Y_{T_{n}} / Y_{0} \cdot P .
$$

By applying assertion (b') to $\bar{X}^{T_{n}}$ (since $\bar{X}^{T_{n}}$ is an $\mathbb{F}$-quasi-left-continuous element of $\left.\mathcal{M}\left(Q_{n}, \mathbb{F}\right)\right)$ and using the fact that $Q_{n}$ is equivalent to $P$, we conclude that $(\phi \cdot$ $\left.\left(X-X^{\tau}\right)\right)^{T_{n}}=\bar{X}^{T_{n}}-\left(\bar{X}^{T_{n}}\right)^{\tau}$ satisfies NUPBR $(\mathbb{G})$. Hence, thanks to Proposition A.4, NUPBR $(\mathbb{G})$ for $X-X^{\tau}$ follows immediately. This ends the proof of $(\mathrm{b}) \Longleftrightarrow$ (c), and that of the proposition as well. 
Remark 2.18 Thanks to [12, Théorème 51, Chapitre III] (see also [17, Theorem 4.26]), if $\mathbb{F}$ is a quasi-left-continuous filtration, then any accessible $\mathbb{F}$-stopping time is predictable and any $\mathbb{F}$-optional process is quasi-left-continuous. Therefore, if we furthermore assume that $\mathbb{F}$ is quasi-left-continuous, then Theorem 2.17 remains valid by substituting assertion (a) by the following assertion

(a') The thin set $\left\{\widetilde{Z}=1>Z_{-}\right\}$is evanescent.

The equivalence between assertion (a') and assertion (b) of Theorem 2.17 remains also valid when we relax the quasi-left-continuous assumption on the filtration $\mathbb{F}$ itself and on $\mathbb{F}$-martingales. This general case, that can be found in [6] and [15], requires more technical arguments.

The enlargement of filtration considered in [8] might appear more general than our current paper. In [8], the authors restrict their attention to the case where $S$ is a continuous $\mathbb{H}$-semimartingale which can be decomposed as

$$
S_{t}=M_{t}+\int_{0}^{t} \eta_{s} d\langle M\rangle_{s}^{\mathbb{H}}, \quad \text { with } \quad \mathbb{E}\left[\int_{0}^{T} \eta_{s}^{2} d\langle M\rangle_{s}^{\mathbb{H}}\right]<\infty
$$

where $M$ is an $\mathbb{H}$-local martingale and $\eta$ is an $\mathbb{H}$-predictable process. Based on the continuity assumption of $S$, we see from [10] that the condition (2.10) is very restrictive compared to NUPBR for $(S, \mathbb{H})$. This is mainly due to the fact that NUPBR for continuous models requires that $\int_{0}^{T} \eta_{s}^{2} d\langle M\rangle_{s}^{\mathbb{H}}$ is finite (almost surely) only, while this random variable is not integrable for many models satisfying NUPBR such as those of [22]. Moreover, for continuous $S$, our current paper presents a very simple approach (see Theorem 2.7), and the true innovation of our work lies in the general case when $S$ has jumps. Note that the condition (2.10) fails for many models. To illustrate this point, we consider a slightly modified example of [10].

Example 2.19 Consider a Poisson process, $N$, with intensity equal to 1 , and a positive real function, $f$, defined on $[0,1]$ that belongs to $L^{1}[0,1] \backslash L^{2}[0,1]$. Let $\Psi, \mu, a$ and $Y$ be those of Example 2.9. Then put $M_{t}:=N_{t}-t$ and

$$
S_{t}:=S_{0}+I_{\left\{Y_{-}>1+a\right\}} \cdot M_{t}-\int_{t_{0}}^{t \vee t_{0}} e^{s} f\left(s-t_{0}\right) I_{\left\{Y_{s-}>1+a\right\}} d s, \quad 0 \leq t \leq T,
$$

where $T:=t_{0}+1$ and $t_{0}:=(1+a) / \mu>0$.

Thus, one can prove that both models $(S, \mathbb{F})$ and $\left(S-S^{\tau}, \mathbb{G}\right)$ fail the condition (2.10). Therefore, this concept can not evaluate the impact of $\tau$ on NUPBR for this context that one can encounter frequently in insurance. Since our results (Theorem 2.12 for instance) are able to deal with any quasi-left-continuous context and any progressive enlargement with an element of $\mathcal{H}$, we conclude that [8] does not cover our results. 


\section{Explicit deflators for a class of $\mathbb{F}$-local martingales}

This section proposes an explicit construction of $\mathbb{G}$-deflator for $M-M^{\tau}$, when $M$ spans the class of $\mathbb{F}$-quasi-left-continuous local martingales orthogonal to $V^{\mathbb{F}}-\left(V^{\mathbb{F}}\right)^{p, \mathbb{F}}$, where $V^{\mathbb{F}}$ is defined in (2.7). The key mathematical idea behind this achievement lies in the exact relationship between the $\mathbb{G}$-compensator and the $\mathbb{F}$-compensator of a process with finite variation when both exist. This is the aim of the first subsection, while the second subsection states results about deflators.

\subsection{Compensators and projections under $\mathbb{G}$ and $\mathbb{F}$}

In the following, we start our study by writing the $\mathbb{G}$-compensators/projections in terms of $\mathbb{F}$-compensators/projections respectively. Although the proofs of the results of this subsection are easy and not technical at all, we opt for delegating them to the Appendix for the reader's convenience.

Lemma 3.1 Suppose that $\tau \in \mathcal{H}$. Then the following assertions hold.

(a) For any $\mathbb{F}$-adapted process $V$ with locally integrable variation, one has

$$
I_{\rrbracket \tau,+\infty \mathbb{I}} \cdot V^{p, \mathbb{G}}=I_{\rrbracket \tau,+\infty \llbracket}\left(1-Z_{-}\right)^{-1} \cdot((1-\widetilde{Z}) \cdot V)^{p, \mathbb{F}},
$$

and on $\rrbracket \tau,+\infty \llbracket$

$$
p, \mathbb{G}(\Delta V)=\left(1-Z_{-}\right)^{-1} p, \mathbb{F}((1-\widetilde{Z}) \Delta V) .
$$

(b) For any $\mathbb{F}$-local martingale $M$, one has, on $\rrbracket \tau,+\infty \rrbracket$

$$
p, \mathbb{G}\left(\frac{\Delta M}{1-\widetilde{Z}}\right)=\frac{p, \mathbb{F}\left(\Delta M I_{\{\widetilde{Z}<1\}}\right)}{1-Z_{-}}, \text {and } p, \mathbb{G}\left(\frac{1}{1-\widetilde{Z}}\right)=\frac{p, \mathbb{F}\left(I_{\{\widetilde{Z}<1\}}\right)}{1-Z_{-}} .
$$

(c) For any quasi-left-continuous $\mathbb{F}$-local martingale $M$, one has

$$
p, \mathbb{G}\left((\Delta M)(1-\widetilde{Z})^{-1} I_{\rrbracket},+\infty \mathbb{I}\right)=0 .
$$

The next lemma focuses on the integrability of the process $(1-\widetilde{Z})^{-1} I_{\rrbracket \tau},+\infty \llbracket$ with respect to any process with $\mathbb{F}$-locally integrable variation. As a result, we complete our comparison of $\mathbb{G}$ and $\mathbb{F}$-compensators.

Lemma 3.2 Let $\tau$ be an honest time and $V$ be a càdlàg and $\mathbb{F}$-adapted process with finite variation. Then the following assertions hold.

(a) The process

$$
U:=(1-\widetilde{Z})^{-1} I_{\rrbracket \tau,+\infty \llbracket} \cdot V
$$

is a well-defined process, i.e., it is $\mathbb{G}$-adapted, càdlàg and has finite variation.

(b) If $V$ belongs to $\mathcal{A}_{\text {loc }}(\mathbb{F})$ (respectively to $\mathcal{A}(\mathbb{F})$ ), then $U \in \mathcal{A}_{\text {loc }}(\mathbb{G})$ (respectively $U \in \mathcal{A}(\mathbb{G})$ ) and

$$
U^{p, \mathbb{G}}=I_{\rrbracket \tau,+\infty \llbracket \mathbb{I}}\left(1-Z_{-}\right)^{-1} \cdot\left(I_{\{\widetilde{Z}<1\}} \cdot V\right)^{p, \mathbb{F}} .
$$


(c) If $\tau$ is a finite honest time, then $I_{\rrbracket \tau,+\infty \llbracket} \cdot V \in \mathcal{A}_{\mathrm{loc}}(\mathbb{G})$ if and only if $(1-\widetilde{Z}) \cdot V$ belongs to $\mathcal{A}_{\mathrm{loc}}(\mathbb{F})$.

(d) If $\tau \in \mathcal{H}$ and $V$ is a nondecreasing and $\mathbb{F}$-predictable process, then for any nonnegative $\mathbb{F}$-predictable process $\varphi$, one has $\varphi I_{\rrbracket} \tau,+\infty \mathbb{I} \cdot V \in \mathcal{A}_{\mathrm{loc}}^{+}(\mathbb{G})$ if and only if $\left(1-Z_{-}\right) \varphi \cdot V \in \mathcal{A}_{\mathrm{loc}}^{+}(\mathbb{F})$, or equivalently if $\varphi I_{\left\{Z_{-}<1\right\}} \cdot V \in \mathcal{A}_{\mathrm{loc}}^{+}(\mathbb{F})$.

\subsection{Construction of deflators}

Herein, we start by introducing a candidate for a deflator as follows.

Proposition 3.3 Suppose that $\tau \in \mathcal{H}$ and consider the $\mathbb{G}$-local martingale

$$
\widehat{m}:=I_{\rrbracket \tau,+\infty \llbracket} \cdot m+\left(1-Z_{-}\right)^{-1} I_{\rrbracket \tau,+\infty \llbracket \mathbb{I}} \cdot\langle m\rangle^{\mathbb{F}},
$$

and the process

$$
W^{\mathbb{G}}:=\left(\left(1-Z_{-}\right)(1-\widetilde{Z})\right)^{-1} I_{\rrbracket},+\infty \mathbb{I} \cdot[m, m],
$$

where $m$ is given in (2.1). Then the following assertions hold.

(a) The nondecreasing and $\mathbb{G}$-optional process $W^{\mathbb{G}}$ belongs to $\mathcal{A}_{\text {loc }}^{+}(\mathbb{G})$.

(b) The $\mathbb{G}$-local martingale

$$
L^{\mathbb{G}}:=\left(1-Z_{-}\right)^{-1} I_{\rrbracket},+\infty \mathbb{I} \cdot \widehat{m}+W^{\mathbb{G}}-\left(W^{\mathbb{G}}\right)^{p, \mathbb{G}},
$$

satisfies the following properties:

(b') $\mathcal{E}\left(L^{\mathbb{G}}\right)>0$ (or equivalently $1+\Delta L^{\mathbb{G}}>0$ ) and $I_{\| 0, \tau \rrbracket} \cdot L^{\mathbb{G}}=0$.

(b") For any quasi-left-continuous $\mathbb{F}$-local martingale $M$, we have

$$
\left[L^{\mathbb{G}}, \widehat{M}\right] \in \mathcal{A}_{\mathrm{loc}}(\mathbb{G}) \text { (i.e., }\left\langle L^{\mathbb{G}}, \widehat{M}\right\rangle^{\mathbb{G}} \text { exists), }
$$

where $\widehat{M}$ is defined via (2.2).

Proof Thanks to Lemma 2.6-(b), $\left(1-Z_{-}\right)^{-1} I_{\rrbracket \tau,+\infty}$ is $\mathbb{G}$-locally bounded. Thus, by combining this fact with $[m, m] \in \mathcal{A}_{\text {loc }}^{+}(\mathbb{F})$ and Lemma 3.2-(b), we conclude that $W^{\mathbb{G}} \in \mathcal{A}_{\text {loc }}^{+}(\mathbb{G})$, and subsequently assertion (a) holds. Thus, the process $L^{\mathbb{G}}$ is a well-defined $\mathbb{G}$-local martingale.

The rest of the proof focuses on proving assertions (b') and (b"). By combining Lemma 3.1-(b), the fact that $\Delta\left(V^{p, \mathbb{H}}\right)=p, \mathbb{H}(\Delta V)$ for any process $V$ with locally integrable variation and any filtration $\mathbb{H}$, and $\Delta m=\widetilde{Z}-Z_{-}$on $\rrbracket \tau,+\infty \llbracket$, we calculate, using (3.2) for the third equality,

$$
\begin{aligned}
& \Delta L^{\mathbb{G}}=\left(1-Z_{-}\right)^{-1} \Delta \widehat{m}+\Delta W^{\mathbb{G}}-\Delta\left(W^{\mathbb{G}}\right)^{p, \mathbb{G}} \\
= & \frac{\Delta m}{1-Z_{-}}+\frac{\Delta\langle m\rangle^{\mathbb{F}}}{\left(1-Z_{-}\right)^{2}}+\frac{(\Delta m)^{2}}{(1-\widetilde{Z})\left(1-Z_{-}\right)}-p, \mathbb{G}\left(\frac{(\Delta m)^{2}}{(1-\widetilde{Z})\left(1-Z_{-}\right)}\right) \\
= & \frac{\Delta m}{1-\widetilde{Z}}+\frac{\Delta\langle m\rangle^{\mathbb{F}}}{\left(1-Z_{-}\right)^{2}}-\frac{p, \mathbb{F}\left((\Delta m)^{2} I_{\{\widetilde{Z}<1\}}\right)}{\left(1-Z_{-}\right)^{2}}=-1+\frac{1-Z_{-}}{1-\widetilde{Z}}+p, \mathbb{F}\left(I_{\{\widetilde{Z}=1\}}\right)
\end{aligned}
$$


Therefore, $1+\Delta L^{\mathbb{G}}=I_{\rrbracket \tau,+\infty \llbracket}\left(\left(1-Z_{-}\right) /(1-\widetilde{Z})+{ }^{p, \mathbb{F}}\left(I_{\{\widetilde{Z}=1\}}\right)\right)+I_{\llbracket 0, \tau \rrbracket}>0$. This proves property (b').

In order to prove property (b"), we consider a quasi-left-continuous $\mathbb{F}$-local martingale $M$. This quasi-left-continuous assumption implies that $\langle m, M\rangle^{\mathbb{F}}$ is continuous and $[X, M] \equiv 0$ for any $\mathbb{G}$-predictable process with finite variation $X$. As a result, we derive

$$
\begin{aligned}
{\left[L^{\mathbb{G}}, \widehat{M}\right] } & =\left[L^{\mathbb{G}}, M-M^{\tau}\right]=\left(1-Z_{-}\right)^{-1} I_{\rrbracket \tau,+\infty \mathbb{I}} \cdot[m, M]+\left[W^{\mathbb{G}}, M\right] \\
& =\frac{1}{1-Z_{-}} I_{\rrbracket \tau,+\infty \mathbb{I}} \cdot[m, M]+\frac{\Delta m}{\left(1-Z_{-}\right)(1-\widetilde{Z})} I_{\rrbracket \tau,+\infty \mathbb{I}} \cdot[m, M] \\
& =(1-\widetilde{Z})^{-1} I_{\rrbracket \tau,+\infty \mathbb{I}} \cdot[m, M] .
\end{aligned}
$$

Therefore, since $[m, M] \in \mathcal{A}_{\text {loc }}(\mathbb{F})$, property (b") follows from combining the above equality and Lemma 3.2-(b). This ends the proof of the proposition.

Below, we elaborate our main results about deflator for "the part-after- $\tau$ ".

Theorem 3.4 Let $\tau \in \mathcal{H}$, and $V^{\mathbb{F}}$ and $L^{\mathbb{G}}$ be defined by (2.7) and (3.6) respectively. If $M$ is a quasi-left-continuous $\mathbb{F}$-local martingale that is orthogonal to $V^{\mathbb{F}}-\left(V^{\mathbb{F}}\right)^{p, \mathbb{F}}$ (i.e., $\sum I_{\left\{\widetilde{Z}=1>Z_{-}\right\}} \Delta M$ is also an $\mathbb{F}$-local martingale), then $\mathcal{E}\left(L^{\mathbb{G}}\right)\left(M-M^{\tau}\right)$ is a $\mathbb{G}$-local martingale.

Proof Suppose that the process $V:=\sum I_{\left\{\widetilde{Z}=1>Z_{-}\right\}} \Delta M$ is an $\mathbb{F}$-local martingale where $M$ is an $\mathbb{F}$-quasi-left-continuous local martingale. Then,

$$
0=\left(\left(1-Z_{-}\right) \cdot V\right)^{p, \mathbb{F}}=\left(I_{\left\{\widetilde{Z}=1>Z_{-}\right\}} \cdot[m, M]\right)^{p, \mathbb{F}} .
$$

Therefore, by combining this equation, (3.7) and Lemma 3.2-(b), we obtain

$$
\begin{aligned}
& M-M^{\tau}+\left\langle L^{\mathbb{G}}, M-M^{\tau}\right\rangle^{\mathbb{G}}=M-M^{\tau}+\left(\frac{I_{\rrbracket},+\infty \mathbb{I}}{1-\widetilde{Z}} \cdot[m, M]\right)^{p, \mathbb{G}} \\
= & M-M^{\tau}+\frac{I_{\rrbracket \tau,+\infty \mathbb{I}}}{1-Z_{-}} \cdot\langle m, M\rangle^{\mathbb{F}}-\frac{I_{\rrbracket},+\infty \mathbb{I}}{1-Z_{-}} \cdot\left(I_{\{\widetilde{Z}=1\}} \cdot[m, M]\right)^{p, \mathbb{F}} \\
= & M-M^{\tau}+\left(1-Z_{-}\right)^{-1} I_{\rrbracket},+\infty \mathbb{I} \cdot\langle m, M\rangle^{\mathbb{F}}=\widehat{M} \in \mathcal{M}_{\mathrm{loc}}(\mathbb{G}) .
\end{aligned}
$$

Thus, the theorem follows immediately from this latter equality combined with integration by parts formula applied to $\left(M-M^{\tau}\right) \mathcal{E}\left(L^{\mathbb{G}}\right)$.

As a consequence of this theorem, we describe a class of $\mathbb{F}$-quasi-left-continuous processes for which NUPBR is preserved for the "part-after- $\tau$ ".

Corollary 3.5 Suppose that $\tau \in \mathcal{H}, S$ is $\mathbb{F}$-quasi-left-continuous, and $L^{\mathbb{G}}$ is given by (3.6). Then the following assertions hold.

(a) $\mathcal{E}\left(L^{\mathbb{G}}\right)\left(M-M^{\tau}\right)$ is a $\mathbb{G}$-local martingale, for any $\mathbb{F}$-quasi-left-continuous $\mathbb{F}$-local martingale, $M$, such that $\left\{\widetilde{Z}=1>Z_{-}\right\} \cap\{\Delta M \neq 0\}$ is evanescent.

(b) If $\left(S, \sum I_{\left\{\widetilde{Z}=1>Z_{-}\right\}} \Delta S\right)$ satisfies $N U P B R(\mathbb{F})$, then $S-S^{\tau}$ satisfies $N U P B R(\mathbb{G})$. 
Proof Assertion (a) follows obviously from Theorem 3.4. The proof of assertion (b) follows immediately from a combination of Theorem 3.4, Proposition A.4 and the fact that for any probability measure $Q$ equivalent to $P$,

$$
\left.Z=1>Z_{-}\right\}=\left\{\widetilde{Z}^{Q}=1>Z_{-}^{Q}\right\}
$$

where $\widetilde{Z}^{Q}$ and $Z^{Q}$ are the $\mathbb{F}$-optional projection under $Q$ of $I_{\llbracket 0, \tau \rrbracket}$ and $I_{\llbracket 0, \tau \llbracket \text { respec- }}$ tively. This last fact is an immediate application of [13, Theorem 86] by taking on the one hand $X=I_{\{\widetilde{Z}=0\}}$ and $Y=I_{\{\widetilde{Z} Q=0\}}$, and on the other hand $X=I_{\left\{Z_{-}=0\right\}}$ and $Y=I_{\left\{Z_{-}^{Q}=0\right\}}$. This ends the proof of the corollary.

We end this section by proving the implication $(b) \Longrightarrow$ (a) of Theorem 2.12 . To this end, we remark that we always have

$$
S-S^{\tau}=\mathcal{T}(S)-(\mathcal{T}(S))^{\tau} \text { and }\{\Delta \mathcal{T}(S) \neq 0\} \cap\left\{\widetilde{Z}=1>Z_{-}\right\}=\emptyset .
$$

Thus, the proof of $(b) \Longrightarrow$ (a) of Theorem 2.12 follows immediately from combining these with Corollary 3.5, Lemma 2.6-(b) and Proposition A.4.

\section{Proof of Theorems 2.12 and 2.15}

This section focuses on the proofs of Theorems 2.12 and 2.15. Both proofs are based essentially on the predictable characteristics of $S$ under $\mathbb{F}$ and $\mathbb{G}$. This section is divided into three subsections. The first subsection recalls the definition of the predictable characteristics, and proposes afterwards a functional $\psi$, which is intimately related to the set $\left\{\widetilde{Z}=1>Z_{-}\right\}$. The functional $\psi$ quantifies the part contained in the latter set which is responsible for the violation of $\operatorname{NUPBR}(\mathbb{G})$. The second and third subsections are devoted to the proofs of Theorems 2.12 and 2.15 respectively.

4.1 Predictable characteristics of $S$ and definition of the functional $\psi$

To the process $S$, we associate $\mu(d t, d x):=\sum_{u>0} I_{\left\{\Delta S_{u} \neq 0\right\}} \delta_{\left(u, \Delta S_{u}\right)}(d t, d x)$ which is the random measure of its jumps. For any nonnegative product-measurable functional $H(t, \omega, x)$, we define the process $H \star \mu$ and a $\sigma$-finite measure $M_{\mu}^{P}$ on the measurable space $\left(\Omega \times \mathbb{R}^{+} \times \mathbb{R}^{d}, \mathcal{F}_{\infty} \otimes \mathcal{B}\left(\mathbb{R}^{+}\right) \otimes \mathcal{B}\left(\mathbb{R}^{d}\right)\right)$ by

$$
H \star \mu_{t}:=\int_{0}^{t} \int_{\mathbb{R}^{d}} H(u, x) \mu(d u, d x) \quad \text { and } M_{\mu}^{P}[H]:=E\left[H \star \mu_{\infty}\right] .
$$

Throughout the rest of the paper, for any filtration $\mathbb{H}$, we denote

$$
\widetilde{\mathcal{O}}(\mathbb{H}):=\mathcal{O}(\mathbb{H}) \otimes \mathcal{B}\left(\mathbb{R}^{d}\right), \quad \widetilde{\mathcal{P}}(\mathbb{H}):=\mathcal{P}(\mathbb{H}) \otimes \mathcal{B}\left(\mathbb{R}^{d}\right),
$$

and $M_{\mu}^{P}[W \mid \widetilde{\mathcal{P}}(\mathbb{H})]$, for a nonnegative or bounded functional $W$, is the unique $\widetilde{\mathcal{P}}(\mathbb{H})$-measurable functional $Y$ satisfying $M_{\mu}^{P}[Y U]=M_{\mu}^{P}[W U]$ for any bounded and $\widetilde{\mathcal{P}}(\mathbb{H})$-measurable functional $U$. The random measure $v(d t, d x)$ is the unique $\mathbb{F}$-predictable 
random measure satisfying $(H \star \mu)^{p, \mathbb{F}}=H \star v$, for any $\widetilde{\mathcal{P}}(\mathbb{F})$-measurable and nonnegative $H$. There is a version of $v$ taking the form of $v(d t, d x)=F_{t}(d x) d A_{t}$ where $A$ is nondecreasing and $\mathbb{F}$-predictable, and $F(d x)$ is an $\mathbb{F}$-predictable kernel. Then the canonical decomposition of $S$ is

$$
S=S_{0}+S^{c}+h \star(\mu-v)+b \cdot A+(x-h) \star \mu,
$$

where $h(x):=x I_{\{|x| \leq 1\}}, S^{c}$ is the continuous $\mathbb{F}$-local martingale part of $S, b$ is an $\mathbb{F}$-predictable process, $h \star(\mu-v)$ is the unique pure jump $\mathbb{F}$-local martingale with jumps given by $h(\Delta S) I_{\{\Delta S \neq 0\}}$, and there exists an $\mathbb{F}$-predictable matrix process, $c$, such that $\left\langle S^{c}, S^{c}\right\rangle^{\mathbb{F}}=c \cdot A$. Then, the quadruplet $(b, c, F, A)$ is the $\mathbb{F}$ - predictable characteristics of $S$.

These characteristics parameterize the model $(S, \mathbb{F}, P)$, and will be used frequently throughout the remaining part of the paper. For more details, we refer to [19, Chapter II] and [17, Chapter XI].

The following identifies explicitly the source of the violation of NUPBR(G) for $S-S^{\tau}$ denoted by the functional $\psi$, and gives some of its properties.

\section{Lemma 4.1 Consider}

$$
f_{m}:=M_{\mu}^{P}[\Delta m \mid \widetilde{\mathcal{P}}(\mathbb{F})] \text {, and } \psi:=M_{\mu}^{P}\left[I_{\{\widetilde{Z}<1\}} \mid \widetilde{\mathcal{P}}(\mathbb{F})\right] \text {. }
$$

Then the following hold.

(a) The process $\left(f_{m}\right)^{2} \star \mu$ belongs to $\mathcal{A}_{\mathrm{loc}}^{+}(\mathbb{F})$, and there exist $\beta_{m} \in \mathcal{L}\left(S^{c}, \mathbb{F}\right)$, i.e., $\left(\beta_{m}\right)^{\mathrm{tr}} c \beta_{m} \cdot A \in \mathcal{A}_{\mathrm{loc}}^{+}(\mathbb{F})$, and $m^{\perp} \in \mathcal{M}_{\mathrm{loc}}(\mathbb{F})$ such that $\left[S^{c}, m^{\perp}\right] \equiv 0$ and

$$
m=m_{0}+\beta_{m} \cdot S^{c}+m^{\perp} .
$$

(b) We have $\{\psi=0\}=\left\{Z_{-}+f_{m}=1\right\} \subset\{\widetilde{Z}=1\}$, $M_{\mu}^{P}$-a.e. or equivalently

$$
\{\psi=0\}=\left\{Z_{-}+f_{m}=1\right\} \subset\{\widetilde{Z}=1\} \quad \text { on }\{\Delta S \neq 0\} .
$$

(c) The nondecreasing process $I_{\left\{\psi=0, Z_{-}<1\right\}} \star \mu$ is càdlàg and $\mathbb{F}$-locally integrable under any probability measure $Q$.

Proof Assertion (a) is proved in [2, Lemma 5.1]. Thus, the remaining proof addresses assertions (b) and (c) in two parts.

Part 1: proof of assertion (b): For any non-negative and $\widetilde{\mathcal{O}}(\mathbb{F})$-measurable functional $W$, we have $E\left[W \star \mu_{\infty}\right]=E\left[M_{\mu}^{P}[W \mid \widetilde{\mathcal{P}}(\mathbb{F})] \star v_{\infty}\right]$. Thus, since $M_{\mu}^{P}[\widetilde{Z} \mid \widetilde{\mathcal{P}}(\mathbb{F})]=Z_{-}+f_{m}$ and $1-\widetilde{Z} \leq I_{\{\widetilde{Z}<1\}}$, we derive

$$
\begin{aligned}
& 0 \leq 1-Z_{-}-f_{m} \leq \psi \quad M_{\mu}^{P} \text {-a.e. and } \\
& E\left[(1-\widetilde{Z}) I_{\left\{Z_{-}+f_{m}=1\right\}} \star \mu_{\infty}\right]=E\left[\left(1-Z_{-}-f_{m}\right) I_{\left\{Z_{-}+f_{m}=1\right\}} \star \mu_{\infty}\right]=0 .
\end{aligned}
$$

These clearly prove that, on the one hand, we have

$$
\{\psi=0\} \subset\left\{Z_{-}+f_{m}=1\right\} \subset\{\widetilde{Z}=1\}, \quad M_{\mu}^{P} \text {-a.e. }
$$

On the other hand, we derive

$$
E\left[I_{\left\{Z_{-}+f_{m}=1\right\}} \psi \star \mu_{\infty}\right]=E\left[I_{\left\{Z_{-}+f_{m}=1\right\}} I_{\{\widetilde{Z}<1\}} \star \mu_{\infty}\right]=0
$$


This proves that $\left\{Z_{-}+f_{m}=1\right\} \subset\{\psi=0\}, M_{\mu}^{P}$-a.e., and the proof of assertion (b) is completed.

Part 2: proof of assertion (c): It follows immediately from combining Lemma 2.11 and the following inequality

$$
H I_{\left\{\psi=0, Z_{-}<1\right\}} \star \mu \leq H I_{\left\{\widetilde{Z}=1>Z_{-}\right\}} \star \mu \leq \sum H I_{\left\{\widetilde{Z}=1>Z_{-}\right\}}=: H \cdot V^{\mathbb{F}},
$$

for $H$ nonnegative and bounded. This ends the proof of the lemma.

We now compute the $\mathbb{G}$-predictable characteristics of $S-S^{\tau}$. Throughout the rest of the paper, we define $\mu^{\mathbb{G}}(d t, d x):=I_{\{t>\tau\}} \mu(d t, d x)$, and deduce that $\nu^{\mathbb{G}}$-its $\mathbb{G}$-compensator- is given by

$$
v^{\mathbb{G}}(d t, d x):=I_{\{t>\tau\}}\left(1-f_{m}(x, t)\left(1-Z_{t-}\right)^{-1}\right) v(d t, d x)
$$

Furthermore, the $\mathbb{G}$-canonical decomposition of $S-S^{\tau}$ is given by

$$
\begin{aligned}
S-S^{\tau}= & \widehat{S^{c}}+h \star\left(\mu^{\mathbb{G}}-v^{\mathbb{G}}\right)+b I_{\rrbracket},+\infty \llbracket \cdot A-\frac{c \beta_{m}}{1-Z_{-}} I_{\rrbracket \tau,+\infty \llbracket} \cdot A \\
& -h \frac{f_{m}}{1-Z_{-}} I_{\rrbracket \tau,+\infty \mathbb{I}} \star v+(x-h) \star \mu^{\mathbb{G}},
\end{aligned}
$$

where $\widehat{S^{c}}$ is defined from $S^{c}$ via (2.2). This decomposition states clearly that the $\mathbb{G}$-predictable characteristics of $S-S^{\tau},\left(b^{\mathbb{G}}, c^{\mathbb{G}}, F^{\mathbb{G}}, A^{\mathbb{G}}\right)$, are given by

$$
\begin{aligned}
b^{\mathbb{G}} & :=b-\left(\int h(x) f_{m}(x) F(d x)+c \beta_{m}\right)\left(1-Z_{-}\right)^{-1} I_{\left\{Z_{-}<1\right\}}, \quad c^{\mathbb{G}}:=c \\
F^{\mathbb{G}}(d x) & :=\left(1-\frac{f_{m}(x)}{1-Z_{-}}\right) I_{\left\{Z_{-}<1\right\}} F(d x), \quad A^{\mathbb{G}}:=A-A^{\tau} .
\end{aligned}
$$

4.2 Proof of the implication (a) $\Longrightarrow$ (b) of Theorem 2.12

This section proves this implication, which is the most difficult and technical part of Theorem 2.12. This proof, done in three steps, relies essentially on applying Theorem A. 3 adequately and repeatedly. The first step is concerned with translating $\operatorname{NUPBR}(\mathbb{G})$ of $S-S^{\tau}$ into the existence of a pair of $\mathbb{F}$-predictable functionals, $\left(\beta^{\mathbb{F}}, f^{\mathbb{F}}\right)$, fulfilling a set of conditions. This is the aim of Lemma 4.2. However, as it will be explained right after the proof of this lemma, this step is not sufficient to conclude NUPBR of $(\mathcal{T}(S), \mathbb{F})$. Thus, the second step, that consists of finding a model $\left(S^{(1)}, \mathbb{F}\right)$ for which NUPBR holds if an only if NUPBR of $(\mathcal{T}(S), \mathbb{F})$ holds also, naturally imposes itself. The core idea and result of this step is stated in Proposition 4.3, where a model $\left(S^{(1)}, \mathbb{F}\right)$ is chosen such that its fulfillment of NUPBR is intimately related to the pair $\left(\beta^{\mathbb{F}}, f^{\mathbb{F}}\right)$ and its set of conditions obtained in step 1 . This fact is proved in step 3 in which the proof of Theorem 2.12 is completed.

Step 1: Herein, we provide an equivalent characterisation for NUPBR of $\left(S-S^{\tau}, \mathbb{G}\right)$, in the spirit of Theorem A.3, but using the filtration $\mathbb{F}$ only. 
Lemma 4.2 Let us define for any $f \in \widetilde{\mathcal{P}}(\mathbb{F})$ and $\alpha>0$, the map $\Phi_{\alpha}$ as

$$
\Phi_{\alpha}(f):=(f-1)^{2} I_{\{|f-1| \leq \alpha\}}+|f-1| I_{\{|f-1|>\alpha\}} .
$$

Then $S-S^{\tau}$ satisfies NUPBR $(\mathbb{G})$ if and only if there exists a pair, $\left(\beta^{\mathbb{F}}, f^{\mathbb{F}}\right)$, of an $\mathbb{F}$-predictable process $\beta^{\mathbb{F}}$ and a $\widetilde{\mathcal{P}}(\mathbb{F})$-predictable functional $f^{\mathbb{F}}$, such that $f^{\mathbb{F}}>0$, $M_{\mu}^{P}$-a.e.,

$$
\left(\beta^{\mathbb{F}}\right)^{\operatorname{tr}} c \beta^{\mathbb{F}} I_{\left\{Z_{-}<1\right\}} \cdot A+\Phi_{\alpha}\left(f^{\mathbb{F}}\right)\left(1-Z_{-}-f_{m}\right) I_{\left\{Z_{-}<1\right\}} \star \mu \in \mathcal{A}_{\text {loc }}^{+}(\mathbb{F}),
$$

and $P \otimes A$-a.e. on $\left\{Z_{-}<1\right\}$,

$$
\begin{aligned}
& \int\left|x f^{\mathbb{F}}(x)\left(1-\frac{f_{m}(x)}{1-Z_{-}}\right)-h(x)\right| F(d x)<+\infty, \\
& b+c\left(\beta^{\mathbb{F}}-\frac{\beta_{m}}{1-Z_{-}}\right)+\int\left(x f^{\mathbb{F}}(x)\left(1-\frac{f_{m}(x)}{1-Z_{-}}\right)-h(x)\right) F(d x) \equiv 0 .
\end{aligned}
$$

Proof In virtue of Theorem A.3, by using the $\mathbb{G}$-predictable characteristics of $S-S^{\tau}$, given in (4.3), we deduce that $S-S^{\tau}$ satisfies NUPBR(G) iff there exists a pair of $\mathbb{G}$-predictable functionals $\left(\beta^{\mathbb{G}}, f^{\mathbb{G}}\right)$ such that $f^{\mathbb{G}}>0$,

$$
\left(\beta^{\mathbb{G}}\right)^{\operatorname{tr}} c \beta^{\mathbb{G}} I_{\rrbracket \tau,+\infty \mathbb{I}} \cdot A+\sqrt{\left(f^{\mathbb{G}}-1\right)^{2} I_{\rrbracket},+\infty \llbracket \star \mu} \in \mathcal{A}_{\mathrm{loc}}^{+}(\mathbb{G}),
$$

and $P \otimes A$-a.e. on $\rrbracket \tau,+\infty \llbracket$,

$$
\begin{aligned}
& \int\left|x f^{\mathbb{G}}(x)-h(x)\right|\left(1-Z_{-}-f_{m}\right) F(d x)<+\infty, \quad \text { and } \\
& 0 \equiv b+c\left(\beta^{\mathbb{G}}-\frac{\beta_{m}}{1-Z_{-}}\right)+\int\left(x f^{\mathbb{G}}(x)\left(1-\frac{f_{m}(x)}{1-Z_{-}}\right)-h(x)\right) F(d x) .
\end{aligned}
$$

Furthermore, given this pair $\left(\beta^{\mathbb{G}}, f^{\mathbb{G}}\right)$, Proposition A.5 guarantees the existence of a pair of $\mathbb{F}$-predictable functionals $\left(\beta^{\mathbb{F}}, f^{\mathbb{F}}\right)$ such that $f^{\mathbb{F}}>0$ and

$$
\left(\beta^{\mathbb{G}}, f^{\mathbb{G}}\right)=\left(\beta^{\mathbb{F}}, f^{\mathbb{F}}\right) \text { on } \rrbracket \tau,+\infty \llbracket \text {. }
$$

Therefore, by inserting (4.11) in the three conditions, (4.8), (4.9) and (4.10), and using afterwards Lemma 3.2-(d) and Proposition B.3 (precisely assertions (b), (c) and (d)), the proof of the lemma follows immediately.

In virtue of Theorem A.3, NUPBR for the model $\left(S^{(0)}, \mathbb{F}\right)$, where

$$
S^{(0)}:=I_{\left\{Z_{-}<1\right\}} \cdot \mathcal{T}(S),
$$

follows from the existence of a pair $\left(\beta^{(0)}, f^{(0)}\right)$ that satisfies (A.3)-(A.5). Thus, the first step to prove NUPBR for $\left(S^{(0)}, \mathbb{F}\right)$ from NUPBR of $\left(S-S^{\tau}, \mathbb{G}\right)$ resides in guessing $\left(\beta^{(0)}, f^{(0)}\right)$ from the pair $\left(\beta^{\mathbb{F}}, f^{\mathbb{F}}\right)$ of Lemma 4.2.

By Lemma A.1, the $\mathbb{F}$-predictable characteristics of $S^{(0)}$ are

$$
\begin{aligned}
b^{(0)} & :=b+\int h(x)(\psi(x)-1) F(d x), \quad c^{(0)}:=c \\
F^{(0)}(d x) & :=\psi(x) F(d x), \quad A^{(0)}:=I_{\left\{Z_{-}<1\right\}} \cdot A .
\end{aligned}
$$


Thus, the pair $\left(\beta^{(0)}, f^{(0)}\right)$ associated to NUPBR of $\left(S^{(0)}, \mathbb{F}\right)$ should satisfy (A.3), (A.4) and -after simplifications using (4.13)-

$$
b+c \beta^{(0)}+\int\left(x f^{(0)}(x) \psi(x)-h(x)\right) F(d x) \equiv 0 .
$$

Therefore, by comparing this equation to (4.7), we conclude that $\left(\beta^{(0)}, f^{(0)}\right)$ and $\left(\beta^{\mathbb{F}}, f^{\mathbb{F}}\right)$ are related as follows.

$\beta^{(0)}:=\left(\beta^{\mathbb{F}}-\frac{\beta_{m}}{1-Z_{-}}\right) I_{\left\{Z_{-}<1\right\}}, f^{(0)}:=f^{\mathbb{F}}(x)\left(1-\frac{f_{m}(x)}{1-Z_{-}}\right) \psi^{-1} I_{\left\{\psi>0, Z_{-}<1\right\}}$.

This (apparently) unique choice for the pair $\left(\beta^{(0)}, f^{(0)}\right)$, that can be derived directly from $\left(\beta^{\mathbb{F}}, f^{\mathbb{F}}\right)$ of Lemma 4.2 , leads to a major obstacle when checking the conditions (A.3) and (A.4) for $\left(\beta^{(0)}, f^{(0)}\right)$. Indeed, we have no information regarding the integrability of $\psi^{-1} I_{\{\psi>0\}}$.

Step 2: In this step, we define an "equivalent" model that will allow us to control this difficult integrability problem. This is the aim of the following.

Proposition 4.3 Let $\mathcal{T}(S)$ be defined in (2.8) and consider

$$
m^{(1)}:=I_{\left\{\psi=0, Z_{-}<1\right\}} \star(\mu-v) \text { and } S^{(1)}:=I_{\left\{Z_{-}<1\right\}} \cdot S-\left[S, m^{(1)}\right] .
$$

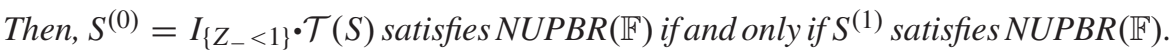

Proof The proof relies essentially on an adequate application(s) of Theorem A.3. To this end, we consider

$$
Z^{(\psi)}:=\mathcal{E}\left(N^{(\psi)}\right) \text { where } N^{(\psi)}:=(\psi-1) I_{\{\psi>0\}} \star(\mu-v) .
$$

Remark that $Z^{(\psi)}$ is a positive $\mathbb{F}$-local martingale and let $\left(T_{n}\right)$ be a sequence of $\mathbb{F}$ stopping times that increases to infinity such that $\left(Z^{(\psi)}\right)^{T_{n}}$ is a u.i. martingale. Define $Q_{n}:=\left(Z^{(\psi)}\right)_{T_{n}} \cdot P$. Hence, in virtue of Proposition A.4, $S^{(1)}$ (resp. $S^{(0)}$ ) satisfied $\operatorname{NUPBR}(\mathbb{F})$ if and only if $\left(\left(S^{(1)}\right)^{T_{n}}, Q_{n}\right)$ (resp. $\left.\left(S^{(0)}\right)^{T_{n}}\right)$ satisfies NUPBR($(\mathbb{F})$ for all $n \geq 1$. Therefore, there is no loss of generality in assuming that $Z^{(\psi)}$ is a uniformly integrable martingale, and hence $Q:=Z_{\infty}^{\psi} \cdot P$ is an equivalent probability measure. Thus, the rest of the proof applies Theorem A.3 to both models $\left(S^{(1)}, Q, \mathbb{F}\right)$ and $\left(S^{(0)}:=I_{\left\{Z_{-}<1\right\}} \cdot \mathcal{T}(S), \mathbb{F}\right)$, and compares the conditions (A.3)-(A.5) associated to these models.

Thanks to Lemma A.2-(b), the $(Q, \mathbb{F})$-predictable characteristics of $S^{(1)}$ are

$$
\begin{aligned}
b^{(1, Q)} & :=b-\int h(x)(\psi(x)-1) F(d x), \quad c^{(1, Q)}:=c, \\
F^{(1, Q)}(d x) & :=\psi(x) F(d x), \quad A^{(1, Q)}:=I_{\left\{Z_{-}<1\right\}} \cdot A .
\end{aligned}
$$

By comparing the above quadruplet to the quadruplet of $\left(S^{(0)}, \mathbb{F}\right)$ given by $(4.13)$, we conclude that both models $\left(S^{(1)}, Q, \mathbb{F}\right)$ and $\left(S^{(0)}, \mathbb{F}\right)$ have the same predictable characteristics. Hence, the proof of the proposition follows immediately from applying Theorem A.3 and using the same pair of $\mathbb{F}$-predictable functionals (i.e., $\left(\beta^{(0)}, f^{(0)}\right)=\left(\beta^{(1, Q)}, f^{(1, Q)}\right)$ ), as the conditions (A.3)-(A.5) are exactly the same for both models. 
Step 3: Herein, we prove that NUPBR for $\left(S-S^{\tau}, \mathbb{G}\right)$ implies NUPBR of $\left(S^{(1)}, \mathbb{F}\right)$, defined in Proposition 4.3, and resume the proof of the implication (a) $\Longrightarrow(b)$ of Theorem 2.12.

Suppose that $S-S^{\tau}$ satisfies NUPBR(G). Then Lemma 4.2 allows us to deduce the existence of $\left(\beta^{\mathbb{F}}, f^{\mathbb{F}}\right)$ satisfying $f^{\mathbb{F}}>0,(4.5),(4.6)$ and (4.7). Consider $\Sigma_{1}:=\left\{Z_{-}<1\right.$ and $\left.\psi>0\right\}$ $\widetilde{\Omega}:=\Omega \times[0,+\infty)$,

$$
\begin{aligned}
\beta^{(1)} & :=\left(\beta^{\mathbb{F}}-\frac{\beta_{m}}{1-Z_{-}}\right) I_{\left\{Z_{-}<1\right\}}, \\
f^{(1)}(x) & :=f^{\mathbb{F}}(x)\left(1-\frac{f_{m}(x)}{1-Z_{-}}\right) I_{\Sigma_{1}}+I_{\widetilde{\Omega} \backslash \Sigma_{1}} .
\end{aligned}
$$

It is obvious that $f^{(1)}>0$. By lemma A.2-(a), the $(P, \mathbb{F})$-predictable characteristics of $S^{(1)}$ are:

$$
\begin{array}{r}
b^{(1)}:=b-\int h(x) I_{\{\psi(x)=0\}} F(d x), \quad c^{(1)}:=c, \\
F^{(1)}(d x):=I_{\{\psi(t, x)>0\}} F(d x), \quad A^{(1)}:=I_{\left\{Z_{-}<1\right\}} \cdot A .
\end{array}
$$

It is clear that, by plugging (4.14) and (4.15) into (4.6) and (4.7), we get

$$
\begin{aligned}
& \int\left|x f^{(1)}(x)-h(x)\right| F^{(1)}(d x)<+\infty \quad P \otimes A^{(1)} \text {-a.e. and } \\
& b^{(1)}+c \beta^{(1)}+\int\left(x f^{(1)}(x)-h(x)\right) F^{(1)}(d x) \equiv 0, \quad P \otimes A^{(1)} \text {-a.e. }
\end{aligned}
$$

Thus, in the rest of this proof, we focus on proving the integrability condition (A.3) for the pair $\left(\beta^{(1)}, f^{(1)}\right)$. Thanks to Proposition B.1-(b), the $\mathbb{F}$-locally boundedness of $\left(1-Z_{-}\right)^{-2} I_{\left\{Z_{-}<1\right\}}$ is guaranteed, and thanks to Lemma 4.1 and (4.5), we have $\left(\beta_{m}\right)^{\operatorname{tr}} c \beta_{m} \cdot A+\left(\beta^{\mathbb{F}}\right)^{\operatorname{tr}} c \beta^{\mathbb{F}} \cdot A^{(1)} \in \mathcal{A}_{\text {loc }}^{+}(\mathbb{F})$. Thus, by combining these two facts, we deduce that $\left(\beta^{(1)}\right)^{\operatorname{tr}} c \beta^{(1)} \cdot A^{(1)} \in \mathcal{A}_{\mathrm{loc}}^{+}(\mathbb{F})$. Therefore, now we focus on proving $\sqrt{\left(f^{(1)}-1\right)^{2} \star \mu^{(1)}} \in \mathcal{A}_{\text {loc }}^{+}(\mathbb{F})$. To this end, we write

$f^{(1)}-1=\left(f^{\mathbb{F}}-1\right)\left(1-\frac{f_{m}(x)}{1-Z_{-}}\right) I_{\Sigma_{1}}-\frac{f_{m}(x)}{1-Z_{-}} I_{\Sigma_{1}}, \quad \Sigma_{1}:=\left\{\psi>0, Z_{-}<1\right\}$.

As a result, since $0 \leq 1-Z_{-}-f_{m} \leq 1$, we obtain

$$
\begin{aligned}
\left(\left(f^{(1)}-1\right)^{2} \star \mu^{(1)}\right)^{\frac{1}{2}} \leq & \left(\frac{\left(f^{\mathbb{F}}-1\right)^{2}\left(1-Z_{-}-f_{m}\right)^{2}}{\left(1-Z_{-}\right)^{2}} \star \mu^{(1)}\right)^{\frac{1}{2}} \\
& +\left(\frac{f_{m}^{2}}{\left(1-Z_{-}\right)^{2}} \star \mu^{(1)}\right)^{\frac{1}{2}}
\end{aligned}
$$

and the right hand side term of the above inequality is smaller than

$$
\begin{aligned}
& \frac{\Phi_{\alpha}\left(f^{\mathbb{F}}\right)}{1-Z_{-}}\left(1-Z_{-}-f_{m}\right) I_{\left\{Z_{-}<1\right\}} \star \mu+\left(\frac{\Phi_{\alpha}\left(f^{\mathbb{F}}\right)}{\left(1-Z_{-}\right)^{2}}\left(1-Z_{-}-f_{m}\right) I_{\left\{Z_{-}<1\right\}} \star \mu\right)^{1 / 2} \\
& \quad+\left(\frac{f_{m}^{2}}{\left(1-Z_{-}\right)^{2}} I_{\left\{Z_{-}<1\right\}} \star \mu\right)^{1 / 2}
\end{aligned}
$$


where $\Phi_{\alpha}($.$) is defined in (4.4). Thus, a combination of this with the local boundedness$ of $\left(1-Z_{-}\right)^{-2} I_{\left\{Z_{-}<1\right\}}$ (guaranteed by Proposition B.1-(b)), (4.5), and $f_{m}^{2} \star \mu \in \mathcal{A}_{\mathrm{loc}}^{+}\left(\mathbb{F}^{*}\right)$ (due to Lemma 4.1)), we conclude that the proof of

$$
\sqrt{\left(f^{(1)}-1\right)^{2} \star \mu^{(1)}}=\sqrt{\left(f^{(1)}-1\right)^{2} I_{\left\{\psi>0, Z_{-}<1\right\} \star \mu}} \in \mathcal{A}_{\mathrm{loc}}^{+}(\mathbb{F})
$$

is completed. This proves that $\left(S^{(1)}, \mathbb{F}\right)$ satisfies NUPBR. Hence by combining this with Proposition 4.3, the proof of (a) $\Longrightarrow$ (b) of Theorem 2.12 follows immediately. This ends the proof of the theorem.

\subsection{Proof of Theorem 2.15}

In virtue of (4.2) and $\rrbracket \tau,+\infty \llbracket \subset\left\{Z_{-}<1\right\}$, the assumption (2.9) holds iff

$$
\begin{aligned}
0 & =E\left[\left(1-Z_{-}\right)^{-1} I_{\left\{Z_{-}+f_{m}=1>Z_{-}\right\}} I_{\rrbracket \tau,+\infty} \star v_{\infty}\right] \\
& =E\left[I_{\left\{Z_{-}+f_{m}=1>Z_{-}\right\}} \star v_{\infty}\right]=E\left[I_{\left\{Z_{-}+f_{m}=1>Z_{-}\right\}} \star \mu_{\infty}\right] .
\end{aligned}
$$

This implies that $I_{\left\{Z_{-}+f_{m}=1>Z_{-}\right\}} \star \nu$ and $I_{\left\{Z_{-}+f_{m}=1>Z_{-}\right\} \star \mu}$ are null. Thus, we deduce that $m^{(1)}=I_{\left\{Z_{-}+f_{m}=1>Z_{-}\right\}} \star \mu-f_{m} I_{\left\{Z_{-}+f_{m}=1>Z_{-}\right\}} \star v$ is also null. Then the proof of the theorem follows immediately from combining this with Proposition 4.3 and Corollary 2.13-(b).

\section{A Predictable characteristics and deflators}

This section conatisn two lemmas, two propositions and a theorem. The two lemmas are new, while most of the rest of the results of this section are elaborated in [2], and we refer the reader to the appendix of that paper for details. Herein, we consider a probability $Q$, a filtration $\mathbb{H}$, and an $(\mathbb{H}, Q)$-quasi-left-continuous semimartingale $X$. To this process, we associate the random measure of its jumps, denoted by $\mu_{X}$, and its $(\mathbb{H}, Q)$-compensator is denoted by $v_{X}$. We suppose that $X$ has the following canonical decomposition

$$
X=X_{0}+X^{c}+h \star\left(\mu_{X}-v_{X}\right)+(x-h) \star \mu_{X}+b \cdot A .
$$

Here $h(x):=x I_{\{|x| \leq 1\}}$ and $h \star\left(\mu_{X}-v_{X}\right)$ represents the unique pure jump (HI, $\left.Q\right)$-local martingale with jumps taking the form of $h(\Delta S) I_{\{\Delta S \neq 0\}}$. We suppose $v_{X}(d t, d x)=F_{t}(d x) d A_{t}$, and $c$ the matrix such that $\left\langle X^{c}\right\rangle^{\mathbb{H}}=c \cdot A$. The quadruplet $(b, c, F, A)$ is the predictable characteristics of $X$ under $(\mathbb{H}, Q)$.

Lemma A.1 The $\mathbb{F}$-predictable characteristics of $S^{(0)}$ defined in (4.12) are

$$
\begin{aligned}
b^{(0)} & :=b+\int h(x)(\psi(x)-1) F(d x), \quad c^{(0)}:=c \\
F^{(0)}(d x) & :=\psi(x) F(d x), \quad A^{(0)}:=I_{\left\{Z_{-}<1\right\}} \cdot A,
\end{aligned}
$$

where $\psi$ is defined in (4.1). 
Proof For the ease of the reader, we recall the canonical decomposition of $S$ under $(\mathbb{F}, P)$

$$
S=S_{0}+S^{c}+h \star(\mu-v)+b \cdot A+(x-h) \star \mu .
$$

Remark that the random measure associated to the jumps of $S^{(0)}$ and its $\mathbb{F}$-compensator are given by

$$
\mu^{(0)}(d t, d x)=I_{\left\{\widetilde{Z}_{t}<1, Z_{t-}<1\right\}} \mu(d t, d x), \quad v^{(0)}(d t, d x)=\psi(x) I_{\left\{Z_{t-}<1\right\}} v(d t, d x) .
$$

Then, it is clear that

$$
I_{\left\{Z_{-}<1\right\}} h \star(\mu-v)=h \star\left(\mu^{(0)}-v^{(0)}\right)+I_{\left\{Z_{-}<1=\widetilde{Z}\right\}} h \star \mu+(\psi-1) h I_{\left\{Z_{-}<1\right\}} \star v .
$$

Therefore, by using the above equation and (A.1), we derive

$$
\begin{aligned}
S^{(0)}= & I_{\left\{Z_{-}<1\right\}} \cdot S-x I_{\left\{Z_{-}<1=\widetilde{Z}\right\}} \star \mu \\
= & I_{\left\{Z_{-}<1\right\}} \cdot S^{c}+h I_{\left\{Z_{-}<1\right\}} \star(\mu-v)+\left(x-h-x I_{\{\widetilde{Z}=1\}}\right) I_{\left\{Z_{-}<1\right\}} \star \mu \\
& +I_{\left\{Z_{-}<1\right\}} b \cdot A \\
= & I_{\left\{Z_{-}<1\right\}} \cdot S^{c}+h \star\left(\mu^{(0)}-v^{(0)}\right)+(x-h) \star \mu^{(0)} \\
& +I_{\left\{Z_{-}<1\right\}}\left(b+\int h(x)(\psi(x)-1) F(d x)\right) \cdot A .
\end{aligned}
$$

As a result, the lemma follows immediately from the above canonical decomposition of $\left(S^{(0)}, \mathbb{F}\right)$.

Lemma A.2 Let $S^{(1)}$ be given in Proposition (4.3). Suppose that the process $Z^{(\psi)}:=\mathcal{E}\left((\psi-1) I_{\{\psi>0\}} \star(\mu-v)\right)$ is a uniformly integrable martingale and define $Q:=Z_{\infty}^{(\psi)} \cdot P$. Then the following assertions hold.

(a) The $(P, \mathbb{F})$-predictable characteristics of $S^{(1)}$ are

$$
\begin{aligned}
b^{(1)} & :=b-\int h(x) I_{\{\psi(x)=0\}} F(d x), \quad c^{(1)}:=c, \\
F^{(1)}(d x) & :=I_{\{\psi(t, x)>0\}} F(d x), \quad A^{(1)}:=I_{\left\{Z_{-}<1\right\}} \cdot A .
\end{aligned}
$$

(b) The $(Q, \mathbb{F})$ predictable characteristics of $S^{(1)}$ are

$$
\begin{aligned}
b^{(1, Q)} & :=b-\int h(x)(\psi(x)-1) F(d x), \quad c^{(1, Q)}:=c, \\
F^{(1, Q)}(d x) & :=\psi(x) F(d x), \quad A^{(1, Q)}:=I_{\left\{Z_{-}<1\right\}} \cdot A .
\end{aligned}
$$

Proof The proof will be achieved in two steps, where we prove assertions (a) and (b) respectively.

Step 1: The random measure of $S^{(1)}$ and its $\mathbb{F}$-compensator are given by

$$
\mu^{(1)}=I_{\left\{\psi>0, Z_{-}<1\right\}} \star \mu, \quad v^{(1)}=I_{\left\{\psi>0, Z_{-}<1\right\}} \star v .
$$

Due to

$$
h I_{\left\{Z_{-}<1\right\}} \star(\mu-v)=h \star\left(\mu^{(1)}-v^{(1)}\right)+h I_{\left\{\psi=0, Z_{-}<1\right\}} \star(\mu-v)
$$


and (A.1), we derive

$$
\begin{aligned}
S^{(1)}= & I_{\left\{Z_{-}<1\right\}} \cdot S-x I_{\left\{\psi=0, Z_{-}<1\right\}} \star \mu \\
= & I_{\left\{Z_{-}<1\right\}} \cdot S^{c}+I_{\left\{Z_{-}<1\right\}} h \star(\mu-v)+I_{\left\{Z_{-}<1\right\}}(x-h) \star \mu \\
& +I_{\left\{Z_{-}<1\right\}} b \cdot A-x I_{\left\{\psi=0, Z_{-}<1\right\}} \star \mu \\
= & I_{\left\{Z_{-}<1\right\}} \cdot S^{c}+h \star\left(\mu{ }^{(1)}-v^{(1)}\right)+h I_{\left\{\psi=0, Z_{-}<1\right\}} \star(\mu-v) \\
& +I_{\left\{Z_{-}<1\right\}}(x-h) \star \mu+I_{\left\{Z_{-}<1\right\}} b \cdot A-x I_{\left\{\psi=0, Z_{-}<1\right\}} \star \mu .
\end{aligned}
$$

After simplifications, we obtain

$$
\begin{aligned}
S^{(1)}= & I_{\left\{Z_{-}<1\right\}} \cdot S^{c}+h \star\left(\mu^{(1)}-v^{(1)}\right)+(x-h) \star \mu^{(1)} \\
& +I_{\left\{Z_{-}<1\right\}}\left(b-\int h(x) I_{\{\psi=0\}} F(d x)\right) \cdot A .
\end{aligned}
$$

Thus, from the above canonical decomposition of $\left(S^{(1)}, \mathbb{F}\right)$, the proof of assertion (a) follows immediately.

Step 2: Since $\mu^{(1)}=I_{\left\{\psi>0, Z_{-}<1\right\}} \star \mu$ (see step 1) for any $H, \widetilde{\mathcal{P}}(\mathbb{F})$-measurable we calculate the $(Q, \mathbb{F})$-compensator of $H \star \mu^{(1)}$ as follows

$$
\begin{aligned}
\left(H \star \mu^{(1)}\right)^{p, Q, \mathbb{F}} & =\left(\left(1+\Delta N^{(\psi)}\right) \cdot\left(H \star \mu^{(1)}\right)\right)^{p, \mathbb{F}} \\
& =\left(\left(1+(\psi-1) I_{\{\psi>0\}}\right) H \star \mu^{(1)}\right)^{p, \mathbb{F}}=\psi H I_{\left\{Z_{-}<1\right\}} \star v,
\end{aligned}
$$

where $N^{(\psi)}:=(\psi-1) I_{\{\psi>0\}} \star(\mu-v)$. We deduce that the $(Q, \mathbb{F})$-compensator of the random measure $\mu^{(1)}$ is given by

$$
v^{(1, Q)}=\psi I_{\left\{Z_{-}<1\right\} \star v}=\psi \star v^{(1)} .
$$

We now remark that

$$
h \star\left(\mu^{(1)}-v^{(1)}\right)=h \star\left(\mu^{(1)}-v^{(1, Q)}\right)+h(\psi-1) \star v^{(1)} .
$$

By inserting this in (A.2), we derive

$$
\begin{aligned}
S^{(0)}= & I_{\left\{Z_{-}<1\right\}} \cdot S^{c}+h \star\left(\mu^{(1)}-v^{(1, Q)}\right)+(x-h) \star \mu^{(1)} \\
& +\left(b-\int h(x) I_{\{\psi(x)=0\}} F(d x)+\int h(x)(\psi(x)-1) I_{\{\psi>0\}} F(d x)\right) I_{\left\{Z_{-}<1\right\}} \cdot A \\
= & I_{\left\{Z_{-}<1\right\}} \cdot S^{c}+h \star\left(\mu^{(1)}-v^{(1, Q)}\right)+(x-h) \star \mu^{(1)} \\
& +\left(b+\int h(x)(\psi(x)-1) F(d x)\right) I_{\left\{Z_{-}<1\right\}} \cdot A .
\end{aligned}
$$

This is the canonical decomposition of $S^{(1)}$ under $(Q, \mathbb{F})$, since $S^{c}$ coincide with $S^{c, Q}$, the continuous local martingale part of $S$ under Q. Therefore, the proof of assertion (b) is achieved and that of the theorem as well. 
Theorem A.3 Let $(X, Q, \mathbb{H})$ be a quasi-left-continuous model, and $(b, c, F, A)$ be its predictable characteristics under $(\mathbb{H}, Q)$. Then $X$ satisfies $N U P B R(\mathbb{H}, Q)$ if and only if there exists a pair $(\beta, f)$ of an $\mathbb{H}$-predictable process $\beta$ and an $\widetilde{\mathcal{P}}(\mathbb{H})$-measurable functional $f$, such that

$$
\begin{aligned}
& f>0, \quad \beta^{\operatorname{tr}} c \beta \cdot A+\sqrt{(f-1)^{2} \star \mu_{X}} \in \mathcal{A}_{\mathrm{loc}}^{+}(\mathbb{H}, Q), \\
& \int|x f(x)-h(x)| F(d x)<+\infty, \quad Q \otimes A-a . e . \\
& b+c \beta+\int(x f(x)-h(x)) F(d x)=0, \quad Q \otimes \text { A-a.e. }
\end{aligned}
$$

See [2, Theorem A.1] for the proof.

Proposition A.4 Let $X$ be an $\mathbb{H}$-semimartingale. Then the following assertions are equivalent.

(a) There exists a sequence $\left(T_{n}\right)_{n \geq 1}$ of $\mathbb{H}$-stopping times that increases to $+\infty$, such that for each $n \geq 1$, there exists a probability $Q_{n}$ on $\left(\Omega, \mathcal{H}_{T_{n}}\right)$ such that $Q_{n}$ is equivalent to $P$ and $X^{T_{n}}$ satisfies $N U P B R(\mathbb{H})$ under $Q_{n}$.

(b) $X$ satisfies $N U P B R(\mathbb{H})$.

(c) There exists an $\mathbb{H}$-predictable process $\phi$, such that $0<\phi \leq 1$ and $(\phi \cdot X)$ satisfies $\operatorname{NUPBR}(\mathbb{H})$.

The proof of this proposition can be found in [2, Proposition 2.5].

Proposition A.5 Suppose that $\tau$ is an honest time, and let $H^{\mathbb{G}}$ be a $\widetilde{\mathcal{P}}(\mathbb{G})$-measurable functional. Then the following assertions hold.

(a) There exist two $\widetilde{\mathcal{P}}(\mathbb{F})$-measurable functionals $H^{\mathbb{F}}$ and $K^{\mathbb{F}}$ such that

$$
H^{\mathbb{G}}(\omega, t, x)=H^{\mathbb{F}}(\omega, t, x) I_{\llbracket 0, \tau \rrbracket}+K^{\mathbb{F}}(\omega, t, x) I_{\rrbracket \tau,+\infty \llbracket} .
$$

(b) If furthermore $H^{\mathbb{G}}>0$ (respectively $H^{\mathbb{G}} \leq 1$ ), then we can choose $K^{\mathbb{F}}>0$ (respectively $K^{\mathbb{F}} \leq 1$ ) in (A.6).

Proof The proofs of assertions (a) and (b) follow from mimicking Jeulin's proof [20, Proposition 5,3], and will be omitted herein.

\section{B $\mathbb{G}$-local integrability versus $\mathbb{F}$-local integrability}

This subsection connects the $\mathbb{G}$-localisation and the $\mathbb{F}$-localisation for the part after $\tau$. This completes the analysis of [2] regarding the issue of local integrability under $\mathbb{F}$ and $\mathbb{G}$, where the part up to $\tau$ is fully discussed. There is a major difference between the current results and those of [2], which lies in the fact that for the case up to $\tau$ we loose information after an $\mathbb{F}$-stopping when we pass from $\mathbb{G}$ to $\mathbb{F}$. However, for the part after $\tau$, as long as $\tau$ is finite, we pass from $\mathbb{G}$-localisation to $\mathbb{F}$-localisation without any loss of information. 
Proposition B.1 The following assertions hold.

(a) If $\tau$ is a finite honest time and $\left(\sigma_{n}^{\mathbb{G}}\right)_{n \geq 1}$ is a sequence of finite $\mathbb{G}$-stopping times that increases to infinity, then there exists a sequence of finite $\mathbb{F}$-stopping times, $\left(\sigma_{n}^{\mathbb{F}}\right)_{n \geq 1}$, that increases to infinity as well and

$$
\max \left(\sigma_{n}^{\mathbb{G}}, \tau\right)=\max \left(\sigma_{n}^{\mathbb{F}}, \tau\right), P \text {-a.s. }
$$

(b) If $\tau \in \mathcal{H}$, then there exists a sequence of $\mathbb{F}$-stopping times, $\left(\sigma_{n}\right)_{n \geq 1}$, that increases to infinity and

$$
\left\{Z_{-}<1\right\} \cap \llbracket 0, \sigma_{n} \rrbracket \subset\left\{1-Z_{-} \geq \frac{1}{n}\right\}, \forall n \geq 1 .
$$

Or equivalently, $\left(1-Z_{-}\right)^{-1} I_{\left\{Z_{-}<1\right\}}$ is $\mathbb{F}$-locally bounded when $\tau \in \mathcal{H}$.

Proof The proof of this proposition is given in two parts where we prove assertions (a) and (b) respectively.

Part 1: The proof of assertion (a) boils down to the following fact:

for any $\mathbb{G}$-stopping time, $\sigma^{\mathbb{G}}$, there exists an $\mathbb{F}$-stopping time, $\sigma^{\mathbb{F}}$ such that

$$
\sigma^{\mathbb{G}} \vee \tau=\sigma^{\mathbb{F}} \vee \tau P \text {-a.s. }
$$

Indeed, if this fact holds, then there exists $\mathbb{F}$-stopping times, $\left(\sigma_{n}\right)_{n \geq 1}$ such that for any $n \geq 1$, the pair $\left(\sigma_{n}^{\mathbb{G}}, \sigma_{n}\right)$ satisfies (B.1). Since $\sigma_{n}^{\mathbb{G}}$ increases with $n$, by putting $\sigma_{n}^{\mathbb{F}}:=\sup _{1 \leq k \leq n} \sigma_{k}$, we can easily prove that the pair $\left(\sigma_{n}^{\mathbb{G}}, \sigma_{n}^{\mathbb{F}}\right)$ satisfies (B.1) as well (this is due to $\max _{1 \leq i \leq n}\left(x_{i} \vee y\right)=\left(\max _{1 \leq i \leq n} x_{i}\right) \vee y$ for any nonnegative numbers $x_{i}, y$ ). Then assertion (a) follows immediately by taking the limit in (B.1) and making use of $\tau<+\infty P$-a.s. which implies that $\sup _{n \geq 1} \sigma_{n}=\lim _{n \rightarrow+\infty} \sigma_{n}^{\mathbb{F}}=+\infty P$-a.s. This shows that the proof of assertion (a) is achieved as long as we prove (B.3). This is the main focus of the rest of this part 1).

By applying Proposition B.2-(c) (given at the end of this proof and is fully due to Barlow [9]) to the process $Y^{\mathbb{G}}:=I_{\llbracket \sigma^{\mathbb{G}} \vee \tau,+\infty \llbracket}$, we obtain the existence of an $\mathbb{F}$-progressively measurable process $K^{\mathbb{F}}$ such that

$$
Y^{\mathbb{G}}=K^{\mathbb{F}} I_{\llbracket \tau,+\infty \llbracket} \cdot
$$

Then put $\sigma:=\inf \left\{t \geq 0: K_{t}^{\mathbb{F}}=1\right\}$. This is an $\mathbb{F}$-stopping time, and due to $\llbracket \sigma^{\mathbb{G}} \vee \tau,+\infty \llbracket \subset\left\{K^{\mathbb{F}}=1\right\}$, we get

$$
\sigma \leq \tau \vee \sigma^{\mathbb{G}} P \text {-a.s. }
$$

By applying Proposition B.2, we deduce the existence of two double sequences of $\mathbb{F}$-stopping times $\left(\alpha_{n m}\right)_{n, m \geq 1}$ and $\left(\beta_{n m}\right)_{n, m \geq 1}$ satisfying the four assertions of Proposition B.2. As a result, we get

$$
\left\{\tau<\sigma^{\mathbb{G}}\right\} \subset \bigcup_{n, m \geq 1}\left\{\tau<\alpha_{n m} \leq \sigma^{\mathbb{G}}<\beta_{n m}\right\} .
$$

Thanks to Proposition B.2-(ii), for $n, m \geq 1$ on $\left\{\alpha_{n m} \leq \sigma^{\mathbb{G}}<\beta_{n m}\right\}, K^{\mathbb{F}}$ is càdlàg and $K^{\mathbb{F}} \equiv 0$ on $\llbracket \tau, \alpha_{n m} \llbracket$. This implies that

$$
\left\{\tau<\alpha_{n m} \leq \sigma^{\mathbb{G}}<\beta_{n m}\right\} \subset\left\{\tau<\alpha_{n m} \leq \sigma \leq \sigma^{\mathbb{G}}<\beta_{n m}\right\} .
$$


By combining this with the fact that $\llbracket \sigma, \sigma+\epsilon \llbracket \cap\left\{K^{\mathbb{F}}=1\right\} \neq \emptyset$ for all $\epsilon>0$, we deduce that

$$
\left\{\tau<\alpha_{n m} \leq \sigma^{\mathbb{G}}<\beta_{n m}\right\} \subset\left\{\tau<\alpha_{n m} \leq \sigma=\sigma^{\mathbb{G}}<\beta_{n m}\right\} \subset\left\{\sigma=\sigma^{\mathbb{G}}\right\} .
$$

A combination of this with (B.5) leads to

$$
\left\{\tau<\sigma^{\mathbb{G}}\right\} \subset\left\{\sigma=\sigma^{\mathbb{G}}\right\} .
$$

Therefore, thanks to this latter inclusion and (B.4), we derive

$$
\begin{aligned}
\tau \vee \sigma^{\mathbb{G}} & =\left(\tau \vee \sigma^{\mathbb{G}}\right) I_{\left\{\sigma^{\mathbb{G}} \leq \tau\right\}}+\left(\tau \vee \sigma^{\mathbb{G}}\right) I_{\left\{\tau<\sigma^{\mathbb{G}}\right\}}=\tau I_{\left\{\sigma^{\mathbb{G}} \leq \tau\right\}}+(\tau \vee \sigma) I_{\left\{\tau<\sigma^{\mathbb{G}}\right\}} \\
& =(\tau \vee \sigma) I_{\left\{\sigma^{\mathbb{G}} \leq \tau\right\}}+(\tau \vee \sigma) I_{\left\{\tau<\sigma^{\mathbb{G}}\right\}}=\tau \vee \sigma .
\end{aligned}
$$

This proves (B.3), and the proof of assertion (a) is completed.

Part 2: Here, we prove assertion (b). Since $\tau \in \mathcal{H}$, then $\left(1-Z_{-}\right)^{-1} I_{\rrbracket},+\infty \llbracket$ is $\mathbb{G}$-locally bounded due to Lemma 2.6-(b). Thus, on the one hand, there exists a sequence of $\mathbb{G}$-stopping times, $\left(\sigma_{n}^{\mathbb{G}}\right)_{n \geq 1}$ that increases to infinity and

$$
\rrbracket \tau,+\infty \llbracket \cap \llbracket 0, \sigma_{n}^{\mathbb{G}} \rrbracket \subset\left\{1-Z_{-} \geq 1 / n\right\} .
$$

On the other hand, thanks to assertion (a), there exists a sequence of $\mathbb{F}$-stopping times, $\left(\sigma_{n}\right)_{n \geq 1}$ that increases to infinity and satisfies (B.1). Then by inserting this in (B.6), we get

$$
\rrbracket \tau,+\infty \llbracket \cap \llbracket 0, \sigma_{n} \rrbracket \subset\left\{1-Z_{-} \geq 1 / n\right\} .
$$

By taking the $\mathbb{F}$-predictable projection on both sides, we get

$$
0 \leq\left(1-Z_{-}\right) I_{\llbracket 0, \sigma_{n} \rrbracket} \leq I_{\left\{1-Z_{-} \geq 1 / n\right\}} .
$$

This implies (B.2). Hence, the proof of assertion (b) is achieved and that of the proposition as well.

Proposition B.2 Suppose that $\tau$ is an honest time. Then the following hold.

(a) There exist two double sequences of $\mathbb{F}$-stopping times $\left(\alpha_{n, m}\right)_{n, m \geq 1}$ and $\left(\beta_{n, m}\right)_{n, m \geq 1}$ such that $\alpha_{n, m} \leq \beta_{n, m} P$-a.s. for all $n, m \geq 1$, and

$$
\rrbracket \tau,+\infty \llbracket \subset\{Z<1\} \subset \bigcup_{n, m \geq 1} \llbracket \alpha_{n, m}, \beta_{n, m} \llbracket .
$$

(b) For any $n, m \geq 1,\left\{\tau \geq \alpha_{n m}\right\} \subset\left\{\tau \geq \beta_{n m}\right\} P$-a.s.

(c) For any $\mathbb{G}$-optional process $Y^{\mathbb{G}}$, there exists an $\mathbb{F}$-progressively measurable process $K^{\mathbb{F}}$ such that

$$
Y^{\mathbb{G}} I_{\llbracket \tau,+\infty \llbracket}=K^{\mathbb{F}} I_{\llbracket \tau,+\infty \llbracket} \cdot
$$

(d) For any $\mathbb{G}$-optional càdlàg process $Y^{\mathbb{G}}$ such that $Y^{\mathbb{G}}=0$ on $\llbracket 0, \alpha_{n, m} \llbracket$ and constant on $\llbracket \beta_{n, m},+\infty \llbracket$, there exists an $\mathbb{F}$-progressively measurable process $K^{\mathbb{F}}$ that is càdlàg and satisfies (B.7).

Proof For the proof we refer the reader to [9]. In fact, assertion (a) is exactly Lemma 4.1-(iv) in [9], while assertion (b) is a combination of Proposition 4.3 and Lemma 4.4-(ii) of the same paper. 
The next result addresses the $\mathbb{G}$-local integrability involving the random measures that is vital for the proof of Theorem 2.12.

Proposition B.3 Suppose that $\tau \in \mathcal{H}$. Let $\Phi_{\alpha}($.$) (for \alpha>0$ ) be defined in (4.4). Then the following properties hold.

(a) Let $f$ be a real-valued and $\widetilde{\mathcal{P}}(\mathbb{H})$-measurable functional. Then $\sqrt{(f-1)^{2} \star \mu}$ belongs to $\mathcal{A}_{\mathrm{loc}}^{+}(\mathbb{H})$ if and only if $\Phi_{\alpha}(f) \star \mu \in \mathcal{A}_{\mathrm{loc}}^{+}(\mathbb{H})$.

(b) Let $f$ be a real-valued and $\widetilde{\mathcal{P}}(\mathbb{H})$-measurable functional. Then $\sqrt{(f-1)^{2} I_{\rrbracket},+\infty \llbracket \star \mu} \in \mathcal{A}_{\mathrm{loc}}^{+}(\mathbb{G})$ iff $\Phi_{\alpha}(f)\left(1-Z_{-}-f_{m}\right) I_{\left\{Z_{-}<1\right\}} \star \mu \in \mathcal{A}_{\mathrm{loc}}^{+}(\mathbb{F})$.

(c) Let $\phi$ be nonnegative and $\mathbb{F}$-predictable process. Then $P \otimes A$-a.e.

$\rrbracket \tau,+\infty \llbracket \subset\{\phi<+\infty\}$ if and only if $\left\{Z_{-}<1\right\} \subset\{\phi<+\infty\}$.

(d) Let $\theta$ be an $\mathbb{F}$-predictable process. Then $P \otimes A$-a.e. $\rrbracket \tau,+\infty \llbracket \subset\{\theta=0\}$

if and only if $\left\{Z_{-}<1\right\} \subset\{\theta=0\}$.

Proof (a) Assertion (a) is borrowed from [2] (see Proposition C.3-(a)).

(b) Thanks to assertion (a), we deduce that $\sqrt{(f-1)^{2} I_{\rrbracket},+\infty \llbracket \star \mu} \in \mathcal{A}_{\text {loc }}^{+}$(G) iff $\Phi_{\alpha}(f) \star \mu^{\mathbb{G}} \in \mathcal{A}_{\text {loc }}^{+}(\mathbb{G})$ iff

$$
\Phi_{\alpha}(f)\left(1-\frac{f_{m}}{1-Z_{-}}\right) I_{\rrbracket \tau,+\infty \llbracket} \star v=\Phi_{\alpha}(f) \star v^{\mathbb{G}} \in \mathcal{A}_{\mathrm{loc}}^{+}(\mathbb{G}) .
$$

Then by directly applying Lemma 3.2-(d) to the pair

$$
(\varphi, V):=\left(\left(1-f_{m}\left(1-Z_{-}\right)^{-1}\right) I_{\left\{Z_{-}<1\right\}}, \Phi_{\alpha}(f) \star \nu\right),
$$

the proof of assertion (b) follows immediately.

(c) Suppose that $P \otimes A$-a.e. that $\rrbracket \tau,+\infty \llbracket \subset\{\phi<+\infty\}$. This is equivalent to $I_{\rrbracket \tau,+\infty \llbracket} \leq I_{\{\phi<+\infty\}} P \otimes A$-a.e.. Then by taking the $\mathbb{F}$-predictable projection on both sides, we get $1-Z_{-} \leq I_{\{\phi<+\infty\}} P \otimes A$-a.e.. This obviously proves that $\rrbracket \tau,+\infty \llbracket \subset\{\phi<+\infty\}$ implies $\left\{Z_{-}<1\right\} \subset\{\phi<+\infty\}$. The reverse sense follows from $\rrbracket \tau,+\infty \llbracket \subset\left\{Z_{-}<1\right\}$. This ends the proof of assertion (c).

(d) The proof of assertion (d) mimics the proof of assertion (c), and will be omitted. This ends the proof of the proposition.

\section{Proofs for Lemmas 3.1 and 3.2 of Sect. 3.1}

Proof of Lemma 3.1 The proof of the lemma will be achieved in three steps.

First step: proof of assertion (a). From Lemma 2.6, the process

$$
I_{\rrbracket \tau,+\infty \llbracket} \cdot V-I_{\rrbracket \tau,+\infty \llbracket} \cdot V^{p, \mathbb{F}}+I_{\rrbracket \tau,+\infty \llbracket}\left(1-Z_{-}\right)^{-1} \cdot\langle V, m\rangle^{\mathbb{F}}
$$

is a $\mathbb{G}$-local martingale, hence

$$
\begin{aligned}
\left(I_{\rrbracket \tau,+\infty \mathbb{I}} \cdot V\right)^{p, \mathbb{G}} & =I_{\rrbracket \tau,+\infty \mathbb{I}} \cdot V^{p, \mathbb{F}}-I_{\rrbracket \tau,+\infty \mathbb{}}\left(1-Z_{-}\right)^{-1} \cdot\langle V, m\rangle^{\mathbb{F}} \\
& =I_{\rrbracket \tau,+\infty \mathbb{I}} \cdot V^{p, \mathbb{F}}-I_{\rrbracket \tau,+\infty \mathbb{}}\left(1-Z_{-}\right)^{-1} \cdot(\Delta m \cdot V)^{p, \mathbb{F}} \\
& =I_{\rrbracket \tau,+\infty \mathbb{I}}\left(1-Z_{-}\right)^{-1} \cdot\left(\left(1-Z_{-}-\Delta m\right) \cdot V\right)^{p, \mathbb{F}}
\end{aligned}
$$


where the second equality follows from Yoeurp's lemma. This ends the proof of (3.1). The equality (3.2) follows immediately from (3.1) by taking the jumps on both sides, and using $\Delta\left(K^{p, \mathbb{H}}\right)=p, \mathbb{H}(\Delta K)$ when both terms exist.

Step 2: proof of assertion (b). By applying (3.2) for $V_{\epsilon, \delta}$ given by

$$
V_{\epsilon, \delta}:=\sum(\Delta M)(1-\widetilde{Z})^{-1} I_{\{|\Delta M| \geq \epsilon, 1-\widetilde{Z} \geq \delta\}} \in \mathcal{A}_{\mathrm{loc}}(\mathbb{F}),
$$

we get, on $\rrbracket \tau,+\infty \llbracket$,

$$
p, \mathbb{G}\left(\Delta M(1-\widetilde{Z})^{-1} I_{\{|\Delta M| \geq \epsilon, 1-\widetilde{Z} \geq \delta\}}\right)=\left(1-Z_{-}\right)^{-1} p, \mathbb{F}\left(I_{\{|\Delta M| \geq \epsilon, 1-\widetilde{Z} \geq \delta\}} \Delta M\right) .
$$

Then the first equality in (3.3) follows by letting $\epsilon$ and $\delta$ go to zero, and we get on $\rrbracket \tau,+\infty \llbracket$

$$
p, \mathbb{G}\left(\frac{\Delta M}{1-\widetilde{Z}}\right)=\left(1-Z_{-}\right)^{-1 p, \mathbb{F}}\left(\Delta M I_{\{1-\widetilde{Z}>0\}}\right)=\left(1-Z_{-}\right)^{-1 p, \mathbb{F}}\left(\Delta M I_{\{\widetilde{Z}<1\}}\right) .
$$

To prove the second equality in (3.3), on $\rrbracket \tau,+\infty \llbracket$ we calculate that,

$$
\begin{aligned}
p, \mathbb{G}\left(\frac{1}{1-\widetilde{Z}}\right) & =\left(1-Z_{-}\right)^{-1}+\left(1-Z_{-}\right)^{-1} p, \mathbb{G}\left(\frac{\Delta m}{1-\widetilde{Z}}\right) \\
& =\left(1-Z_{-}\right)^{-1}+\left(1-Z_{-}\right)^{-2} p, \mathbb{F}\left(\Delta m I_{\{1-\widetilde{Z}>0\}}\right) \\
& =\left(1-Z_{-}\right)^{-1} p, \mathbb{F}\left(I_{\{\widetilde{Z}<1\}}\right) .
\end{aligned}
$$

The second equality is due to (3.2), and the third equality follows from combining $p, \mathbb{F}(\Delta m)=0$, and $\Delta m=\widetilde{Z}-Z_{-}$. This proves assertion (b).

Step 3: proof of assertion (c). The proof of (3.4) follows immediately from assertion (b) and the fact that the thin process $p, \mathbb{F}\left(I_{\{\widetilde{Z}<1\}} \Delta M\right)$ may take nonzero values on countably many predictable stopping times only, on which $\Delta M$ already vanishes. This completes the proof of the lemma.

Proof Lemma 3.2 The proof of this lemma is given in three parts. In the first part we prove both assertions (a) and (b), while in the second and the third parts we focus on assertions (c) and (d) respectively.

Part 1: Let $V$ be an $\mathbb{F}$-adapted process with finite variation, and denote by $\operatorname{Var}(V)$ its variation. Then we obtain

$$
\operatorname{Var}(U)=(1-\widetilde{Z})^{-1} I_{\rrbracket \tau,+\infty \mathbb{I}} \cdot \operatorname{Var}(V) .
$$

Therefore, since $1-\widetilde{Z}_{t}=P\left[\tau<t \mid \mathcal{F}_{t}\right] \leq 1-Z_{t}$, for any bounded nonnegative and $\mathbb{F}$-optional process $\phi$ such that $\phi \cdot \operatorname{Var}(V) \in \mathcal{A}^{+}(\mathbb{F})$, we obtain

$$
\begin{aligned}
& E\left[(\phi \cdot \operatorname{Var}(U))_{\infty}\right]=E\left[\int_{0}^{\infty} \frac{\phi_{t} I_{\{t>\tau\}}}{1-Z_{t}} d \operatorname{Var}(V)_{t}\right] \\
= & E\left[\int_{0}^{\infty} \frac{\phi_{t} P\left[\tau<t \mid \mathcal{F}_{t}\right]}{1-Z_{t}} I_{\left\{Z_{t}<1\right\}} d \operatorname{Var}(V)_{t}\right] \leq E\left[(\phi \cdot \operatorname{Var}(V))_{\infty}\right] .
\end{aligned}
$$

As a result, by taking $\phi=I_{\llbracket 0, \sigma \llbracket}$ in (C.1), for an $\mathbb{F}$-stopping time $\sigma$ such that $\operatorname{Var}(V)^{\sigma-} \in \mathcal{A}^{+}(\mathbb{F})$, we get $E\left[\operatorname{Var}(U)_{\sigma-}\right] \leq E\left[\operatorname{Var}(V)_{\sigma_{-}}\right]$. This proves that the 
process $U$ has a finite variation and hence is well defined as well. It is clear that $U$ is $\mathbb{G}$-adapted, while being càdlàg follows immediately from (C.1). This ends the proof of assertion (a).

To prove assertion (b), we assume that $V \in \mathcal{A}_{\mathrm{loc}}(\mathbb{F})$ and consider $\left(\vartheta_{n}\right)_{n \geq 1}$, a sequence of $\mathbb{F}$-stopping times that increases to $+\infty$ such that $\operatorname{Var}(V)^{\vartheta_{n}}$ belongs to $\mathcal{A}^{+}(\mathbb{F})$. Then by choosing $\phi=I_{\llbracket 0, \vartheta_{n} \rrbracket}$ in $(\mathrm{C} .1)$, we conclude that $U$ belongs to $\mathcal{A}_{\text {loc }}(\mathbb{G})$ whenever $V$ does under $\mathbb{F}$. For the case when $V \in \mathcal{A}(\mathbb{G})$, it is enough to take $\phi=1$ in (C.1), and conclude that $U \in \mathcal{A}(\mathbb{G})$. To prove (3.5), for any $n \geq 1$, we put

$$
U_{n}:=(1-\widetilde{Z})^{-1} I_{\rrbracket \tau,+\infty \llbracket} I_{\left\{\widetilde{Z}_{\left.\leq 1-\frac{1}{n}\right\}}\right.} \cdot V, \quad n \geq 1 .
$$

Then thanks to (3.1), we derive

$$
U^{p, \mathbb{G}}=\lim _{n \longrightarrow+\infty}\left(U_{n}\right)^{p, \mathbb{G}}=\lim _{n \longrightarrow+\infty}\left(1-Z_{-}\right)^{-1} I_{\rrbracket \tau,+\infty \mathbb{I}} \cdot\left(I_{\left\{\widetilde{Z}_{\leq 1}-\frac{1}{n}\right\}} \cdot V\right)^{p, \mathbb{F}} .
$$

This clearly implies (3.5).

Part 2: It is enough to prove the assertion for the case when $V$ is nondecreasing. Thus, suppose that $V$ is nondecreasing. It follows that $(1-\widetilde{Z}) \cdot V \in \mathcal{A}_{\text {loc }}^{+}(\mathbb{F})$ implies $I_{\rrbracket \tau,+\infty \llbracket} \cdot V \in \mathcal{A}_{\text {loc }}^{+}(\mathbb{G})$. Hence, for the rest of this part, we focus on proving the reverse. Suppose $I_{\rrbracket} \tau,+\infty \mathbb{I} \cdot V \in \mathcal{A}_{\text {loc }}^{+}(\mathbb{G})$. Then there exists a sequence $\mathbb{G}$-stopping times $\left(\sigma_{n}^{\mathbb{G}}\right)$ that increases to infinity and $\left(I_{\rrbracket \tau,+\infty} \cdot V\right)^{\sigma_{n}^{\mathbb{G}}} \in \mathcal{A}^{+}(\mathbb{G})$. Thanks to Proposition B.1(a), we obtain a sequence of $\mathbb{F}$-stopping times, $\left(\sigma_{n}^{\mathbb{F}}\right)_{n \geq 1}$, that increases to infinity and $\sigma_{n}^{\mathbb{G}} \vee \tau=\tau \vee \sigma_{n}^{\mathbb{F}}$. Therefore, we get $\left(I_{\rrbracket},+\infty \mathbb{I} \cdot V\right)^{\sigma_{n}^{\mathbb{G}}} \equiv\left(I_{\rrbracket},+\infty \mathbb{I} \cdot V\right)^{\sigma_{n}^{\mathbb{F}}}$ and hence

$$
E\left[(1-\widetilde{Z}) \cdot V_{\sigma_{n}^{\mathbb{F}}}\right]=E\left[I_{\rrbracket \tau,+\infty \mathbb{I}} \cdot V_{\sigma_{n}^{\mathbb{F}}}\right]=E\left[I_{\rrbracket \tau,+\infty \mathbb{I}} \cdot V_{\sigma_{n}^{\mathbb{G}}}\right]<+\infty .
$$

This proves that the process $(1-\widetilde{Z}) \cdot V$ belongs to $\mathcal{A}_{\text {loc }}^{+}(\mathbb{F})$, and the proof of assertion (c) is achieved.

Part 3: The proof of assertion (d) follows all the steps of the proof of assertion (c), except (C.2) takes the form of

$$
E\left[\left(1-Z_{-}\right) \varphi \cdot V_{\sigma_{n}^{\mathbb{F}}}\right]=E\left[I_{\rrbracket},+\infty \llbracket \varphi \cdot V_{\sigma_{n}^{\mathbb{F}}}\right]=E\left[I_{\rrbracket},+\infty \llbracket \varphi \cdot V_{\sigma_{n}^{\mathbb{G}}}\right]<+\infty
$$

instead due to the predictability of $V$. This proves that $I_{\rrbracket \tau,+\infty \llbracket \varphi} \varphi V \in \mathcal{A}_{\text {loc }}^{+}(\mathbb{G})$ if and only if $\left(1-Z_{-}\right) \varphi \cdot V \in \mathcal{A}_{\mathrm{loc}}^{+}(\mathbb{F})$, while the equivalence $\left(1-Z_{-}\right) \varphi \cdot V \in \mathcal{A}_{\mathrm{loc}}^{+}(\mathbb{F})$ if and only if $I_{\left\{Z_{-}<1\right\}} \varphi \cdot V \in \mathcal{A}_{\text {loc }}^{+}(\mathbb{F})$ follows from the $\mathbb{F}$-locally boundedness of $\left(1-Z_{-}\right)^{p} I_{\left\{Z_{-}<1\right\}}$ for any real number $p$ (see Proposition B.1-(b) for details). This ends the proof of assertion (d) and the proof of the lemma as well.

Acknowledgements The research of Tahir Choulli and Jun Deng is supported financially by the Natural Sciences and Engineering Research Council of Canada, through NSERC RGPIN 04987. The research of Anna Aksamit and Monique Jeanblanc is supported by Chaire Markets in transition, (Fédération Bancaire Française) and ILB, Labex ANR 11-LABX-0019.

The authors are very grateful to two anonymous referees, an anonymous associate editor, and Martin Schweizer for their valuable and helpful comments/suggestions that helped improved this paper tremendously. 


\section{References}

1. Acciaio, B., Fontana, C., Kardaras, C.: Arbitrage of the first kind and filtration enlargements in semimartingale financial models. Stochastic Processes and their Applications, 126, 1761-1784, (2016).

2. Aksamit, A., Choulli, T., Deng, J., Jeanblanc, M.: No-Arbitrage up to random horizon for quasi-left-continuous models. Finance and Stochastics, forthcoming, (2017). Available online at http://arxiv.org/abs/1310.1142

3. Aksamit, A., Choulli, T., Deng, J., Jeanblanc, M.: Arbitrages in a progressive enlargement setting. In: C. Hillairet et al. (eds.), Arbitrage, Credit and Informational Risks, volume 5, Peking Univ. Ser. Math., pages 53-86, World Sci. Publ. (2014).

4. Aksamit, A., Choulli, T., Jeanblanc, M.: On an optional semimartingale decomposition and the existence of a deflator in an enlarged filtration. In memoriam Marc Yor. In: C.D. Martin et al. (eds.), Séminaire de Probabilités XLVII, Vol. 2137, Lecture Notes in Math., pages 187-218, Springer, (2015).

5. Aksamit, A., Choulli, T., Jeanblanc, M.: Classification of random time and applications. Working paper, (2015). Available online at https://arxiv.org/abs/1605.03905

6. Aksamit, A.: Random times, enlargement of filtration and arbitrages, PhD Thesis, Evry-Val d'Essonne University, (2014).

7. Ansel, J.-P., Stricker, C.: Couverture des actifs contingents. Ann. Inst. Henri Poincaré 30, 303-315, (1994).

8. Ankirchner, S., Dereich, S., Imkeller, P.: The Shannon information of filtrations and the additional logarithmic utility of insiders. Annals of Probability, 34, 743-778, (2006).

9. Barlow, M. T.: Study of a filtration expanded to include an honest time. Probability theory and related fields, 44, 307-323, (1978).

10. Choulli, T., Stricker, C.: Deux applications de la décomposition de Galtchouk-Kunita-Watanabe. In: J. Azéma, et al. (eds.), Séminaire de Probabilités XXX, Vol. 1626, Lecture Notes in Math., Springer (1996).

11. Choulli T., Deng, J. Ma, J.: How non-arbitrage, viability and numéraire portfolio are related. Finance and Stochastics, 19, 719-741, (2015).

12. Dellacherie, C.: Capacités et processus stochastiques, Springer, (1972).

13. Dellacherie, C., Meyer, P-A.: Probabilités et Potentiel, chapitres V-VIII,Hermann, Paris, (1980), English translation : Probabilities and Potentiel, chapters V-VIII, North-Holland, (1982).

14. Dellacherie, M., Maisonneuve, B. and Meyer, P-A.: Probabilités et Potentiel, chapitres XVII-XXIV: Processus de Markov (fin), Compléments de calcul stochastique, Hermann, Paris (1992).

15. Deng J.: Essays on Arbitrage Theory for a Class of Informational Markets, PhD. Thesis, University of Alberta (2014).

16. Fontana, C., Jeanblanc, M., Song, S.: On arbitrages arising with honest times. Finance and Stochastics 18, 515-543, (2014).

17. He, S. W., Wang, J. G., Yan, J. A.: Semimartingale theory and stochastic calculus. CRC Press (1992).

18. Imkeller, P.: Random times at which insiders can have free lunches, Stochastics and Stochastic Reports , 74, 465-487, (2002).

19. Jacod, J., Shiryaev, A.N.: Limit Theorems for Stochastic Processes, Springer Verlag, 2nd Edition, (2003).

20. Jeulin, T.: Semi-martingales et Grossissement d'une Filtration. In: A. Dold and B. Eckmann (eds.), Lecture Notes in Mathematics, Vol. 833, Springer, (1980).

21. Kardaras, C.: Market viability via absence of arbitrage of the first kind. Finance and Stochastics, 16 , 651-667, (2012).

22. Loewenstein, M., Willard, G. A.: Local martingales, arbitrage, and viability free snacks and cheap thrills. Economic Theory, 16, 135-161, (2000).

23. Platen, E.: A benchmark approach to finance, Mathematical Finance, 16, 131-151, (2006).

24. Takaoka, K., Schweizer, M.: A note on the condition of no unbounded profit with bounded risk. Finance and Stochastics, 18, 393-405, (2014). 